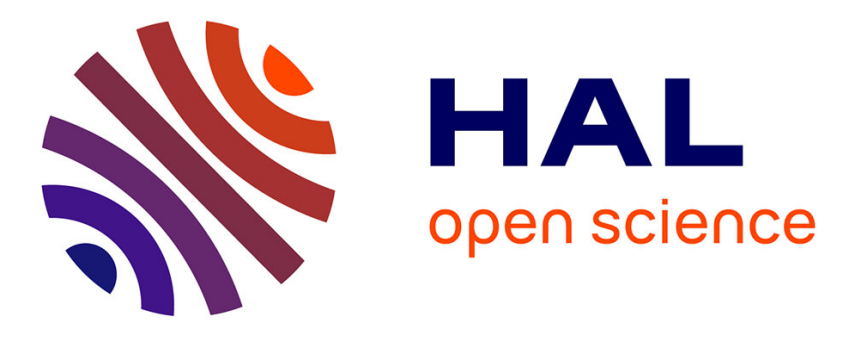

\title{
Detection and localization of hydromechanical disturbances in a sandbox using the self-potential method
}

\author{
A. Crespy, A. Revil, N. Linde, S. Byrdina, Abderrahim Jardani, A. Boleve, P \\ Henry
}

\section{To cite this version:}

A. Crespy, A. Revil, N. Linde, S. Byrdina, Abderrahim Jardani, et al.. Detection and localization of hydromechanical disturbances in a sandbox using the self-potential method. Journal of Geophysical Research : Solid Earth, 2008, 113, pp.B01205. 10.1016/j.sedgeo.2007.09.002 . hal-00485357

\section{HAL Id: hal-00485357 https://hal.science/hal-00485357}

Submitted on 8 Dec 2020

HAL is a multi-disciplinary open access archive for the deposit and dissemination of scientific research documents, whether they are published or not. The documents may come from teaching and research institutions in France or abroad, or from public or private research centers.
L'archive ouverte pluridisciplinaire HAL, est destinée au dépôt et à la diffusion de documents scientifiques de niveau recherche, publiés ou non, émanant des établissements d'enseignement et de recherche français ou étrangers, des laboratoires publics ou privés. 


\title{
Detection and localization of hydromechanical disturbances in a sandbox using the self-potential method
}

\author{
A. Crespy, ${ }^{1}$ A. Revil,${ }^{2,8,9}$ N. Linde,${ }^{3}$ S. Byrdina, ${ }^{4}$ A. Jardani, ${ }^{2,5,6}$ A. Bolève, ${ }^{7,8}$ \\ and P. Henry ${ }^{10}$ \\ Received 12 March 2007; revised 8 September 2007; accepted 5 November 2007; published 30 January 2008.
}

[1] Four sandbox experiments were performed to understand the self-potential response to hydro-mechanical disturbances in a water-infiltrated controlled sandbox. In the first two experiments, $\sim 0.5 \mathrm{~mL}$ of water was abruptly injected through a small capillary at a depth of $15 \mathrm{~cm}$ using a syringe impacted by a hammer stroke. In the second series of experiments, $\sim 0.5 \mathrm{~mL}$ of pore water was quickly pumped out of the tank, at the same depth, using a syringe. In both type of experiments, the resulting self-potential signals were measured using 32 sintered $\mathrm{Ag} / \mathrm{AgCl}$ medical electrodes. In two experiments, these electrodes were located $3 \mathrm{~cm}$ below the top surface of the tank. In two other experiments, they were placed along a vertical section crossing the position of the capillary. These electrodes were connected to a voltmeter with a sensitivity of $0.1 \mu \mathrm{V}$ and an acquisition frequency of $1.024 \mathrm{kHz}$. The injected/pumped volumes of water produced hydromechanical disturbances in the sandbox. In turn, these disturbances generated dipolar electrical anomalies of electrokinetic nature with an amplitude of few microvolts. The source function is the product of the dipolar Green's function by a source intensity function that depends solely on the product of the streaming potential coupling coefficient of the sand to the pore fluid overpressure with respect to the hydrostatic pressure. Numerical modeling using a finite element code was performed to solve the coupled hydro-mechanical problem and to determine the distribution of the resulting self-potential during the course of these experiments. We use 2D and 3D algorithms based on the cross-correlation method and wavelet analysis of potential fields to show that the source was a vertical dipole. These methods were also used to localize the position of the source of the hydromechanical disturbance from the self-potential signals recorded at the top surface of the tank. The position of the source agrees with the position of the inlet/outlet of the capillary showing the usefulness of these methods for application to active volcanoes.

Citation: Crespy, A., A. Revil, N. Linde, S. Byrdina, A. Jardani, A. Bolève, and P. Henry (2008), Detection and localization of hydromechanical disturbances in a sandbox using the self-potential method, J. Geophys. Res., 113, B01205, doi:10.1029/2007JB005042.

\section{Introduction}

[2] Distinct electromagnetic (EM) signals are generated during rock fracturing, steam injection in Hot Dry Rock (HDR) reservoirs, detonation of explosive charges in boreholes, and generally speaking any kind of hydro-mechanical disturbances affecting the porous and conductive ground [e.g., Surkov and Pilipenko, 1997; Ushijima et al., 1999; Yoshida, 2001; Gaffet et al., 2003; Yoshida and Ogawa,

\footnotetext{
${ }^{1}$ CNRS-CEREGE, Université Aix Marseille III, Aix en Provence, France.

${ }^{2}$ Colorado School of Mines, Department of Geophysics, Golden, Colorado, USA.

${ }^{3}$ Swiss Federal Institute of Technology, Institute of Geophysics, Zurich, Switzerland.

${ }^{4}$ IPGP, 4 Place Jussieu, Paris, France.

Copyright 2008 by the American Geophysical Union. 0148-0227/08/2007JB005042\$09.00
}

2004; Soloviev and Sweeney, 2005; Moore and Glaser, 2006, 2007; Park et al., 2007]. In addition, possible EM signals in various frequency ranges have been reported preceding earthquakes [e.g., Tate and Daily, 1989; FraserSmith et al., 1990; Dea et al., 1991; Park et al., 1993, Fenoglio et al., 1995, and references therein]. The selfpotential method consists in passively recording the fluctuations of the electrical at the surface of the Earth or in boreholes to evidence polarization mechanisms occurring at depth. Byrdina et al. [2003] observed well-defined time

\footnotetext{
${ }^{5}$ Laboratoire de Géologie, Faculté des Sciences, Université de Rouen, Mont Saint Aignan, France.

${ }^{6}$ Bureau d'Etude ALISE, Saint Jacques-sur-Darnétal, France.

${ }^{7}$ SOBESOL, Savoie Technolac, BP 230, F-73375 Le Bourget du Lac Cedex, France.

${ }^{8}$ LGIT (UMR 5559), University of Savoie, Equipe Volcan, Chambéry, France.

${ }^{9}$ GdR FORPRO 0788, CNRS-ANDRA, France.

${ }^{10}$ Collège de France, Université Aix Marseille III, France.
} 
variations of the self-potential correlated with ultra long period seismic signals associated with ongoing fracturing at the Merapi volcano. Self-potential signals have also been observed in geothermal fields [e.g., Ishido, 1981, 1989; Revil and Pezard, 1998; Revil et al., 1999a, 1999b; Yasukawa et al., 2002].

[3] Understanding of these EM disturbances could be used to develop new geophysical methods to monitor fracturing and hydro-mechanical disturbances in real time in the Earth to a depth of few kilometers. These signals could be also very important in the growing field of $\mathrm{CO}_{2}$ sequestration to monitor in real-time the migration of $\mathrm{CO}_{2}$ in a sedimentary reservoir or the rupturing of a pressure seal [e.g., Moore et al., 2004]. Similar researches are presently carried out in biomechanics [Garon et al., 2002], in the study of deformation of glacier by Earth tides [Kulessa et $a l ., 2003]$, and in the study of deformation and leakage in Earth dams [Wilt and Corwin, 1989; Titov et al., 2000; Sheffer and Howie, 2003].

[4] The main challenges in the study of self-potential signals associated with hydromechanical disturbances are (1) to understand the physics underlying these phenomena and (2) to propose mathematical algorithms to locate the source of these EM disturbances in the ground. It is also important to validate these algorithms against experimental data recorded in well-controlled conditions [Minsley et al., 2007]. However, observations of self-potential signals corresponding to deformation were probably not available before because of the sensitivity of the apparatus used. Most apparatus used in geophysics have a sensitivity of $0.1 \mathrm{mV}$. In the present work, we use a highly sensitive voltmeter with sintered $\mathrm{Ag} / \mathrm{AgCl}$ electrodes with a sensitivity better than $0.1 \mu \mathrm{V}$.

[5] There are, at least, three physical phenomena that could be responsible for electromagnetic signals during fracturing and hydro-mechanical disturbances. They are the piezoelectric effect [e.g., Nitsan, 1977; Yoshida et al., 1994, 1997]; the migration of mobile electronic charge carriers activated by stress drops [Freund et al., 2006a, 2006b]; and the electrokinetic effect [e.g., Fedorov et al., 2001; Yoshida and Ogawa, 2004; Moore and Glaser, 2006; Revil and Linde, 2006]. However, it seems unlikely that the piezoelectric effect and the migration of mobile electronic charge carriers activated by stress drops can be responsible for observed self-potential signals in the shallow Earth since the magnitude of these two phenomena are much smaller than the electrokinetic effect [see Nitsan, 1977; Yoshidaet al., 1994, 1997; Moore and Glaser, 2007]. Yoshida et al. [1994, 1997] and Moore and Glaser [2007] provided convincing arguments that the electrokinetic effect is the most likely physical mechanism susceptible to produce large EM signals in the first kilometers of the Earth crust. In a porous pack of sand grains, the piezoelectric effect can be ruled out as generating any electromagnetic effects because the quartz crystals are randomly oriented. At depths greater than a few kilometers, the migration of mobile electronic charge carriers activated by stress drops could be the main current-driving mechanism [Freund et al., 2006a, 2006b].

[6] In this paper, we are interested in performing wellconstrained sandbox experiments for the purpose of understanding the electrostatic signature associated with hydro-mechanical disturbances caused by a sudden change in fluid pressure in a water-saturated controlled sandbox. To our knowledge, this is the first time that such type of experiment is undertaken. A very sensitive voltmeter with a high acquisition frequency and connected to very small ( $\sim 1 \mathrm{~mm}$ ) non-polarizing silver/silver chloride electrodes was used. This novel system was initially developed in the context of electro-encephalography. It has a sensitivity 3 orders of magnitude better than previous systems used for similar experiments in geophysics [see Butler et al., 1990; Perrier et al., 1997, 1998; Perrier and Morat, 2000; Rizzo et al., 2004].

[7] In addition, an analytical model and finite element numerical simulations were used to simulate the sandbox experiments. These approaches were based on the model of self-potential signals developed recently by Revil and Linde [2006] for electroporoelastic media.

[8] Finally, we applied wavelet analysis to the selfpotential field to demonstrate that the source, in the initial stage of the experiments, was equivalent to a vertical dipole. We also used a cross-correlation method between the selfpotential field (normalized by the power of the signal) and the Green's function of a dipole to retrieve the position of the hydro-mechanical disturbance in the sandbox.

\section{Electrokinetic Coupling}

[9] The surface of any mineral in contact with water is charged. This charge is attached to the grains. This fixed charge comprises the surface sites (e.g., silanol and aluminol surface groups) plus the counterions sorbed onto the surface. A complete description of this electrical double layer is given by Lorne et al. [1999], Revil and Leroy [2001], Leroy and Revil [2004], and Leroy et al. [2007] and will not be repeated here. This fixed charge is counterbalanced by mobile charges located in the vicinity of the surface of the grains, inside the water phase, in the so-called electrical diffuse layer [Gouy, 1910]. The drag of the mobile electrical charge of the electrical diffuse layer by the flow of the pore water causes a net current density $\mathbf{J}_{\mathrm{S}}$ (expressed in $\mathrm{A} \mathrm{m}^{-2}$ ) [e.g., Haartsen and Toksöz, 1996; Block and Harris, 2006]. This current source density represents the net charge passing per unit surface area of a cross-section of the porous material and per unit time. To our knowledge, the first rigorous attempt to combine the theory of self-potential to poroelastic deformation of a porous material was made by Chandler [1981]. Chandler [1981] was interested by using the self-potential method to probe the existence of Biot's slow wave. The formulation we adopted below is quite different from the classical formulation based on the socalled zeta potential and the pressure field. Following Revil [2007], Revil et al. [2005], Crespy et al. [2007], Revil and Linde [2006], and Linde et al. [2007], our formulation of the coupled hydroelectric problem will be based on the excess of charge per unit pore volume and the seepage velocity.

[10] Neglecting electro-osmosis in the macroscopic momentum equation [e.g., Sill, 1983], the constitutive equations for $\mathbf{J}$ (in $\mathrm{A} \mathrm{m}^{-2}$ ) and the Darcy velocity $\mathbf{U}$ (in $\mathrm{m} \mathrm{s}^{-1}$ ) are given by [e.g., Sill, 1983],

$$
\begin{gathered}
\mathbf{J}=-\sigma \nabla \psi+\mathbf{J}_{s}, \\
\mathbf{U}=-\frac{k}{\eta_{f}} \nabla p,
\end{gathered}
$$


where $\mathbf{J}_{\mathrm{S}}$ is the streaming current density (the subscript "s" stands for the fact that this current density is a source current density), $p$ (in $\mathrm{Pa}$ ) is the pore fluid pressure, $\psi($ in $\mathrm{V}$ ) is the electrical potential, $\sigma$ (in $\mathrm{S} \mathrm{m}^{-1}$ ) is the electrical conductivity, $k\left(\right.$ in $\left.\mathrm{m}^{2}\right)$ is the permeability of the porous medium, and $\eta_{f}$ (in Pa s) is the dynamic viscosity of the pore water. The continuity equations for the charge and the mass of the pore water are [e.g., Sill, 1983],

$$
\begin{gathered}
\nabla \cdot \mathbf{J}=0, \\
\nabla \cdot\left(\rho_{f} \mathbf{U}\right)=-\frac{\partial}{\partial t}\left(\rho_{f} \phi\right),
\end{gathered}
$$

where $\rho_{f}$ is the density of the pore water (in $\mathrm{kg} \mathrm{m}^{-3}$ ), and $\phi$ the porosity $\left(m_{f}=\rho_{f} \phi\right.$ is the mass of the pore water per unit volume of the porous material).

[11] Using Biot's theory, the continuity equation for the mass of the pore fluid in a deforming porous medium can be written in term of the bulk deformation $\varepsilon$ of the porous material and time variation of the fluid pressure by [Palciauskas and Domenico, 1989],

$$
\begin{gathered}
\nabla \cdot\left(\rho_{f} \mathbf{U}\right)=-\rho_{f}\left[\xi \frac{d \varepsilon}{d t}+\left(\frac{1}{R}-\frac{\xi}{H}\right) \frac{d p}{d t}\right], \\
\frac{1}{R}-\frac{\xi}{H}=\frac{1}{\rho_{f}}\left(\frac{\partial m_{f}}{\partial p}\right)_{\varepsilon} \\
\frac{1}{H}=\frac{1}{\rho_{f}}\left(\frac{\partial m_{f}}{\partial \sigma}\right)_{p} \\
\frac{1}{R}=\frac{1}{\rho_{f}}\left(\frac{\partial m_{f}}{\partial p}\right)_{\sigma}
\end{gathered}
$$

where $\varepsilon$ is the trace of the deformation tensor, $\sigma$ is the mean stress (in $\mathrm{Pa}$ ) and $\varepsilon$ is the bulk deformation of the medium. The coefficients $R, \xi$, and $H$ are the so-called Biot coefficient of linear poro-elasticity (all expressed in $\mathrm{Pa}$ ). The coefficient $1 / R$ represents a measure of the change in water content for a given change in pore fluid pressure when the porous material is permitted to drain freely. The coefficient $(1 / R-\xi / H)$ represents a measure of the amount of water which can be forced into a porous material under pressure while the volume of the material is kept constant. The coefficient $1 / H$ represents a measure of the change in water content for a given change in confining stress when the material is permitted to drain freely. Despite the fact that these equations were initially derived to model poro-elastic deformation, they can also be used in the case of irreversible deformations as shown by Palciauskas and Domenico [1989].

[12] According to the model developed by Revil et al. [2005] [see also Crespy et al., 2007; Revil and Linde, 2006; Linde et al., 2007], the source current density is given by $\mathbf{J}_{\mathrm{S}}=$ $\bar{Q}_{V} \mathbf{U}$ where $\bar{Q}_{V}$ is the excess of charge of the diffuse layer per unit pore volume (in $\mathrm{C} \mathrm{m}^{-3}$ ). An alternative and more classical approach is to express the source current density in terms of pressure gradient $\mathbf{J}_{S}=-L \nabla p$ [e.g., Ishido and Mizutani, 1981; Sill, 1983; Haartsen and Toksöz, 1996; Block and Harris, 2006; Jardani et al., 2006] where $L$ is the streaming current coupling coefficient. In all cases, the streaming potential coupling coefficient is defined by $\mathrm{C}=$ $(\partial \psi / \partial p)_{\mathbf{J}=0}$. With the model developed by Revil and Linde [2006], an explicit relationship between the streaming potential coupling coefficient and the excess charge density of the pore water is $C=-\bar{Q}_{\nu} k /\left(\eta_{f} \sigma\right)$. In the classical approach, the streaming potential coupling coefficient is given by the Helmholtz-Smoluchowski equation or a similar equation [e.g., Pengra and Wong, 1995; Szymczyk et al., 2002].

[13] Combining equations (1) and (3) yields a generalized Poisson equation for the self-potential $\psi$ (expressed in V),

$$
\nabla \cdot(\sigma \nabla \psi)=\Im,
$$

where $\Im$ is the volumetric current density (in $\mathrm{A} \mathrm{m}^{-3}$ ) given by,

$$
\begin{gathered}
\Im=\nabla \cdot \mathbf{J}_{S} \\
\Im=\bar{Q}_{V} \nabla \cdot \mathbf{U}+\nabla \bar{Q}_{V} \cdot \mathbf{U} \\
\Im=-\bar{Q}_{V} \xi \frac{d \varepsilon}{d t}-\bar{Q}_{V}\left(\frac{1}{R}-\frac{\xi}{H}\right) \frac{d p}{d t}+\nabla \bar{Q}_{V} \cdot \mathbf{U}
\end{gathered}
$$

where equation (5) has been used for a slightly compressible pore fluid. According to equation (12), there are three electrokinetic sources terms able to generate self-potential signals in a deforming water-saturated porous medium. The first contribution results from the bulk deformation $d \varepsilon / d t$ of the porous body. If the medium has incompressible grains, we have $d \varepsilon / d t=[1 /(1-\phi)] d \phi / d t$. If follows that if the medium is affected by dilatancy $(d \varepsilon / d t \geq 0)$, the first contribution $\Im_{1}=-\bar{Q}_{V} \xi d \varepsilon / d t$ is negative because $\bar{Q}_{V}$ is generally positive (see Revil et al. [2005] for clay minerals and Linde et al. [2007] for sand). The second contribution, $\Im_{2}=-\bar{Q}_{V}(1 / R-\xi / H) d p / d t$, results from time variation of the fluid pressure. In areas where the fluid pressure increases over time, this contribution is negative. The third contribution $\Im_{3}=\nabla \bar{Q}_{V} \cdot \mathbf{U}$ is related to flow of the pore water in domains characterized by a gradient of the volumetric charge density $\bar{Q}_{V}$. As shown below, this contribution plays a key-role during a pulse injection/ pumping of water.

\section{Sandbox Experiments}

[14] Four sandbox experiments were carried out to observe and analyze the electrical signals resulting from the pulse injection or pumping of water in a controlled sandbox. The experiments were performed in an unshielded room using a glass tank to limit electrostatic effects. The tank was open at the top and partially filled with a well-sorted sand mixed with an electrolyte. 

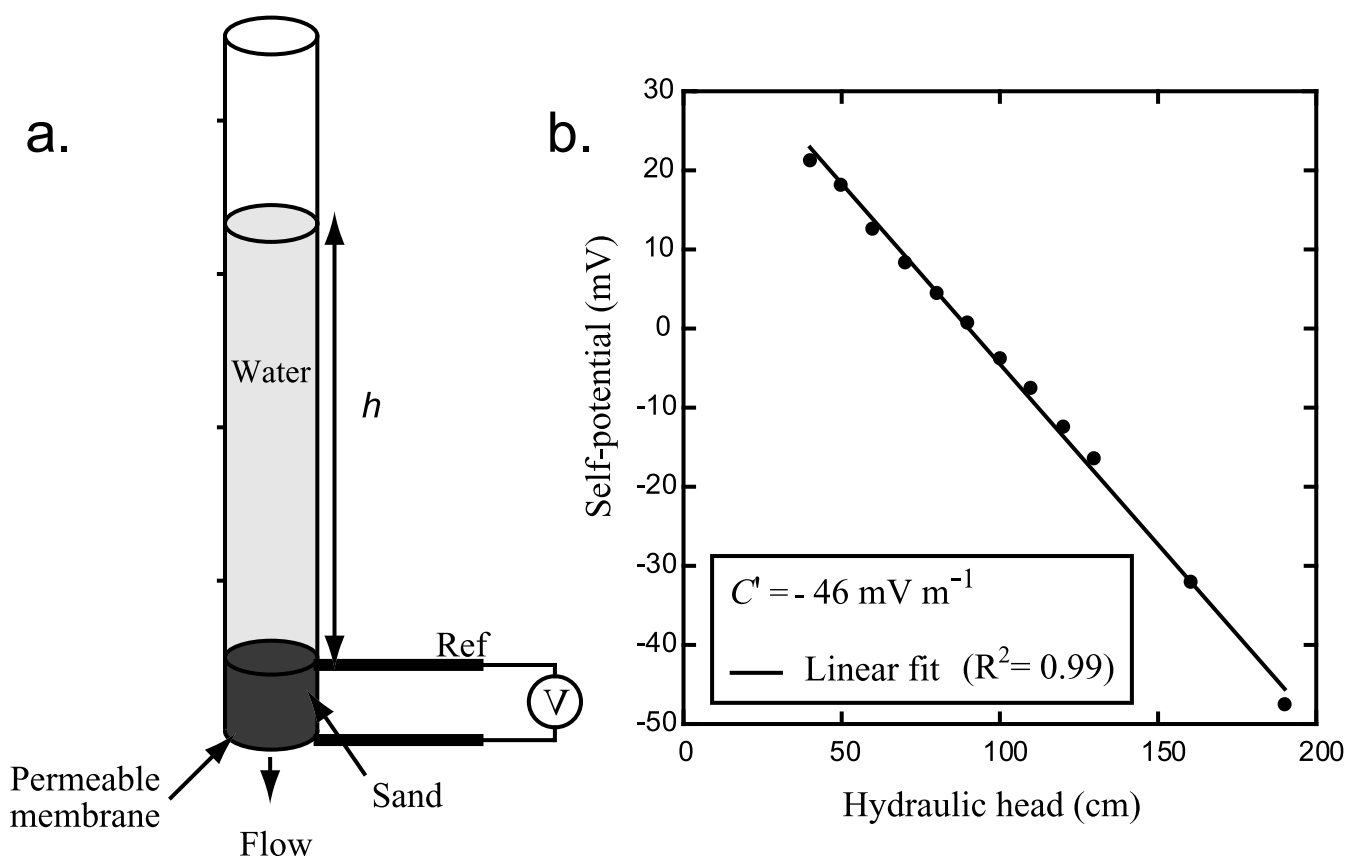

Figure 1. Experimental determination of the streaming voltage coupling coefficient of the sand filling the tank. (a) Experimental setup showing the sample located at the bottom of a Plexiglas tube. The electrical potential difference at the end-faces of the sample (called the streaming potential) is recorded with a calibrated Metrix MX-20 voltmeter and two non-polarizing $\mathrm{Ag} / \mathrm{AgCl}_{2}$ electrodes. (b) Measured streaming potentials versus the imposed hydraulic heads. The streaming potential coupling coefficient corresponds to the slope of the linear trend.

\subsection{Properties of the Sand}

[15] The sandbox was partially filled with a well-calibrated silica sand. The grain diameters were in the range 100 $160 \mu \mathrm{m}$ with a mean diameter of $132 \mu \mathrm{m}$ and a standard deviation of $20 \mu \mathrm{m}$. From X-ray diffraction, the sand was composed mainly of silica $\mathrm{SiO}_{2}(\approx 95 \%), \mathrm{KSi}_{3} \mathrm{AlO}_{8}(4 \%)$, and $\mathrm{NaAlSi}_{3} \mathrm{O}_{8}(<1 \%)$. The porosity of the compacted sand was $\phi=0.34 \pm 0.02$. Steady state flow in a permeameter yielded a mean permeability $k=7.3 \times 10^{-12} \mathrm{~m}^{2}$ (the hydraulic conductivity $K$ was therefore equal to $7.1 \times$ $10^{-5} \mathrm{~m} \mathrm{~s}^{-1}$ with a dynamic viscosity $\eta_{f}$ of $10^{-3} \mathrm{~Pa} \mathrm{~s}$ at $20^{\circ} \mathrm{C}$ ). The electrical formation factor $F$ determined from electrical conductivity measurements with brine solutions $(\mathrm{NaCl})$ at various salinities was equal to $4.3 \pm 0.1$ [see Suski et al., 2004].

[16] The streaming potential coupling coefficient of the sand was determined using the same experimental setup of Suski et al. [2006]. All the experiments reported in the present paper were made with an electrolyte composed of sodium hypochlorite $(\mathrm{NaClO})$ with an electrical conductivity $\sigma_{f}=5.610^{-3} \mathrm{~S} \mathrm{~m}^{-1}$ at $25^{\circ} \mathrm{C}$ and a $\mathrm{pH}$ of 6.8 . This electrolyte was used to avoid the growth of bacteria in the tank. The coupling coefficient determined using the experimental setup shown in Figure 1 was $-46 \pm 2 \mathrm{mV} \mathrm{m}^{-1}$. The conductivity of the saturated sand was equal to $1.31 \times 10^{-3} \mathrm{~S}$ $\mathrm{m}^{-1}$. The streaming current coupling coefficient was therefore $L=-C \sigma=5.6 \times 10^{-9} \mathrm{~A}(\mathrm{~Pa} \mathrm{~m})^{-1}$. Using $C=-\bar{Q}_{V} k /$ $\left(\eta_{f} \sigma\right.$ ) (see section 2) and $\eta_{f}=10^{-3} \mathrm{~Pa} \mathrm{~s}$, we obtain $\bar{Q}_{V}=0.77 \mathrm{C}$ $\mathrm{m}^{-3}$. This value is very similar to the value obtained independently by Linde et al. [2007] $\left(\bar{Q}_{V}=0.48 \mathrm{C} \mathrm{m}^{-3}\right)$, who studied the primary drainage of a sand column using the same sand, but saturated by tap water rather than the solution described above.

\subsection{The Voltmeter and the Active Electrodes}

[17] The self potential signals were monitored with 32 sintered $\mathrm{Ag} / \mathrm{AgCl}$ electrodes developed for electroencephalography, that is for the study of electrical signals at the surface of the scalp due to polarization processes occurring in the brain. By integrating an amplifier with the sintered $\mathrm{Ag}-\mathrm{AgCl}$ electrode, extremely low-noise measurements free of interference are possible (see www.biosemi.com). These active electrodes have very low output impedance and therefore all problems with regards to capacitive coupling between the cable and sources of interference are eliminated. Using this approach, noise levels as low as the thermal noise level of the electrode impedance were achieved. These electrodes are also water-proof and their size is close to $1 \mathrm{~mm}$. This small size (with respect to the size of the array) implies that these electrodes can be treated as point measurements.

[18] In the two first experiments (termed Experiments \#1 and \#2 below), these electrodes were regularly distributed at $3 \mathrm{~cm}$ below the top surface of the tank (see Figure 2) and connected to the ActiveTwo voltmeter manufactured by Biosemi. This voltmeter has 280 channels, a DC amplifier, and 24-bit resolution (see www.biosemi.com). This voltmeter has a resolution of $\sim 0.1 \mu \mathrm{V}$, a maximum frequency of acquisition of $2.048 \mathrm{kHz}$, a bandwidth from $\mathrm{DC}$ to $7 \mathrm{kHz}$, and an input impedance for the electrode of $300 \mathrm{MOhm}$ at $50 \mathrm{~Hz}$. In the third and fourth experiments (Experiments \#3, and \#4), the electrodes were placed on a vertical plane, so it was possible to sample the variations of the electrical 


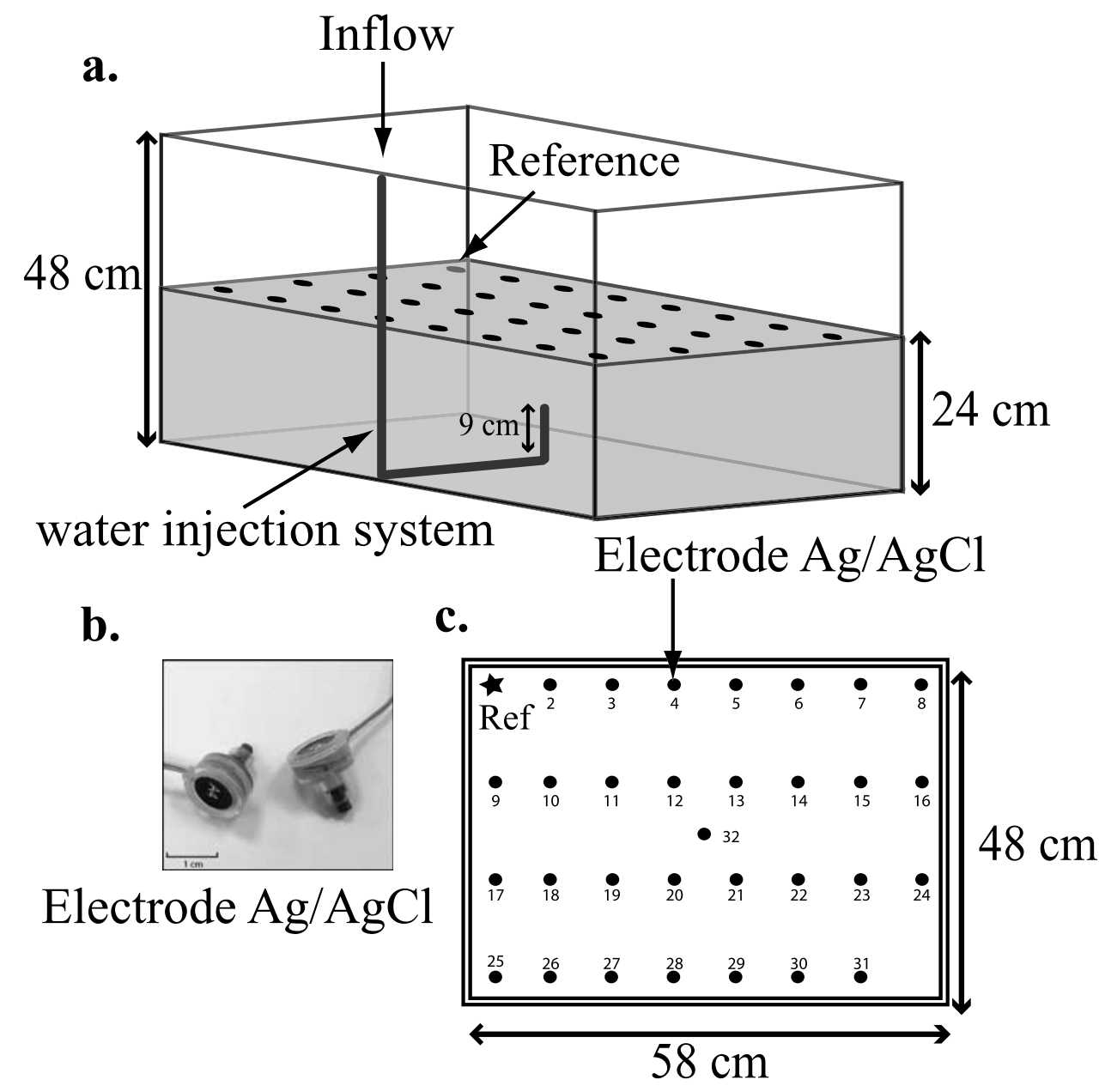

Figure 2. Sketch of the geometry of the sandbox experiment. A total of 32 non-polarizing electrodes are located near the top surface of the tank, which is partially filled with a well-calibrated sand and saturated by a solution of known composition and electrical conductivity. (a) Geometry of the tank. (b) Picture of the self-potential sintered $\mathrm{Ag} / \mathrm{AgCl}$ electrodes developed by BioSemi. These electrodes are very sensitive thanks to a built in amplifier. (c) Sketch of the top surface of the tank showing the position of the electrodes in the vicinity of the top surface of the tank. The electrodes are located at a depth of $3 \mathrm{~cm}$. "Ref" indicates the position of the reference electrode. Electrode \#32 is located just above the inlet/outlet of the capillary.

potential around the end of the capillary. The characteristics of the ActiveTwo voltmeter are much better than the characteristic of the multichannel used usually in the field or in the laboratory for self-potential monitoring and which have a sensitivity on the order of $0.1 \mathrm{mV}$ [see Perrier et al., 1997, 1998; Perrier and Morat, 2000; Rizzo et al., 2004; Maineult et al., 2005; Suski et al., 2006].

\subsection{Injection Experiment}

[19] For experiment \#1, we injected $\sim 0.5 \mathrm{~mL}$ of solution in the central part of the tank at a depth of $15 \mathrm{~cm}$. The water was injected through a relatively rigid capillary filled with water and connected to a Syria syringe. Injection was done by the impact of a hammer stroke on the syringe end-face. The diameter of the capillary was $2 \mathrm{~mm}$. It was ensured that the injected water had the same chemical composition as the water in the tank by extracting the water directly from the tank a few minutes before the experiment. In this way, we avoided any membrane/diffusion polarization effects that could be associated with a difference of the chemical potential between the injected solution and the pore water filling the sand [e.g., Maineult et al., 2005, 2006a, 2006b; Revil et al., 2005]. The self-potential response was measured with a sampling frequency of $1.024 \mathrm{kHz}$. The selfpotential data were referenced and transformed to the frequency domain using a Fast Fourier Transform (FFT). We observed that the $50 \mathrm{~Hz}$ (and harmonics) of the anthropic electrical current strongly dominated the raw self-potential data. In order to remove these components of the self-potential time series, the signals were low-pass filtered with a cut-off frequency of $30 \mathrm{~Hz}$ and an attenuation of 5 decades at $50 \mathrm{~Hz}$. The filtered signals were then backtransformed to the time domain [see also Rizzo et al., 2004].

[20] Snapshots of the filtered self-potential distribution at the surface of the tank are shown on Figure 3. These data exhibit a well-defined negative self-potential anomaly with a maximum amplitude of $-6 \mu \mathrm{V}$. This anomaly is centered right above the injection point. We observed a change in the 
a.

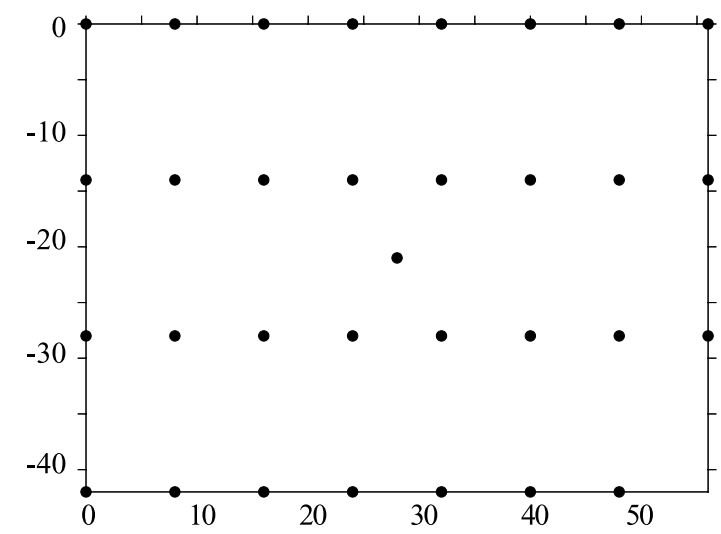

b.

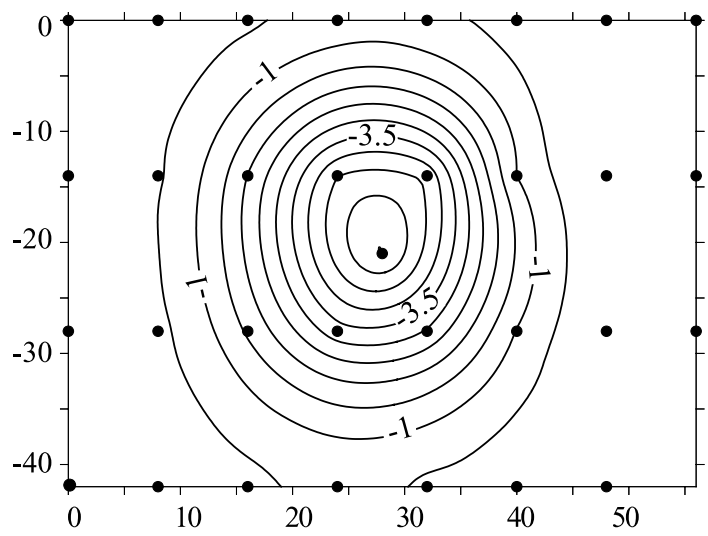

c.

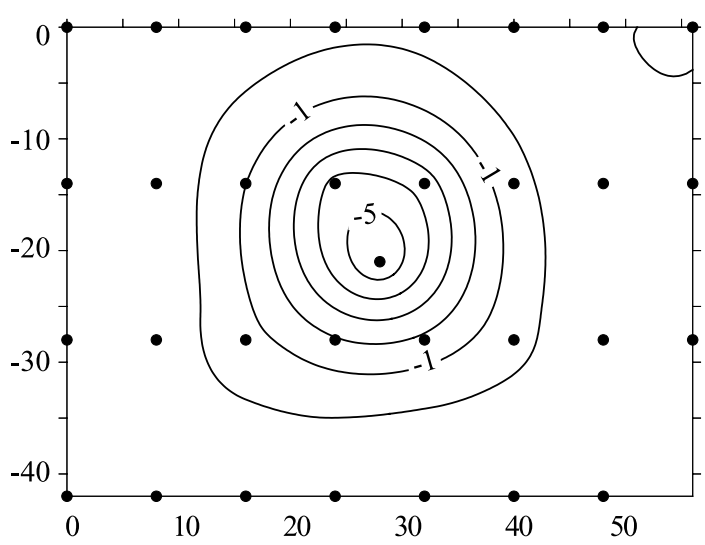

\section{Self-potential (in $\mu \mathrm{V}$ )}

d.

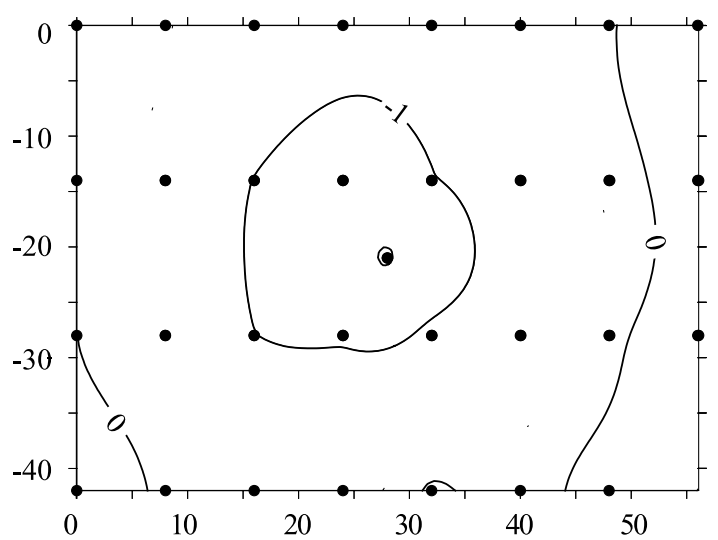

e.

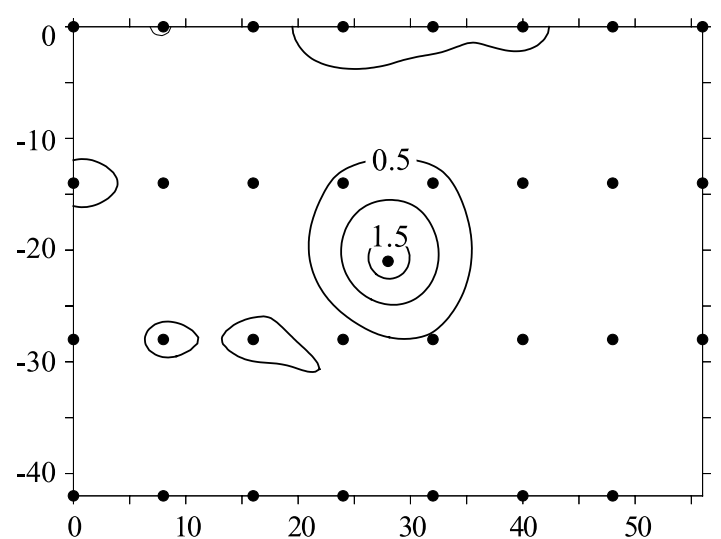

f.

$\mathrm{t}_{4}=1.9 \mathrm{~s}$

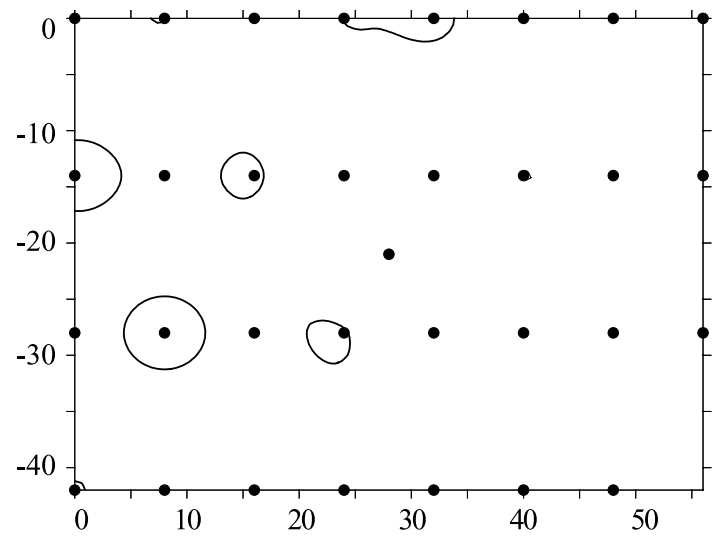

Figure 3. Time-lapse distribution of the self-potential measured at a depth of $3 \mathrm{~cm}$ below the ground surface ( $t=0$ corresponds to the injection of the pressure pulse, Experiment \#1). Note the change of polarity over time in the self-potential distribution. At $t=1.9 \mathrm{~s}$, the self-potential anomaly has vanished.

polarity of the self-potential anomaly $0.35 \mathrm{~s}$ after the time of the injection (Figure 4). However, this experiment was repeated several times and the reversal of the self-potential signal was found to be non-reproducible if the shock of the hammer on the syringe is softer than for Experiment \#1 (see an example in section 3.5 below).

\subsection{Pumping Test Experiment}

[21] A second experiment was performed by quickly pumping out of the tank a prescribed amount of water $(\sim 0.5 \mathrm{~mL})$ from the capillary (Experiment \#2). This was done by hand and by using the same syringe as previously. A rough estimate of the pumping rate is $2.0 \pm 0.5 \mathrm{~mL} \mathrm{~s}^{-1}$. The filtered data reveal a positive self-potential anomaly 


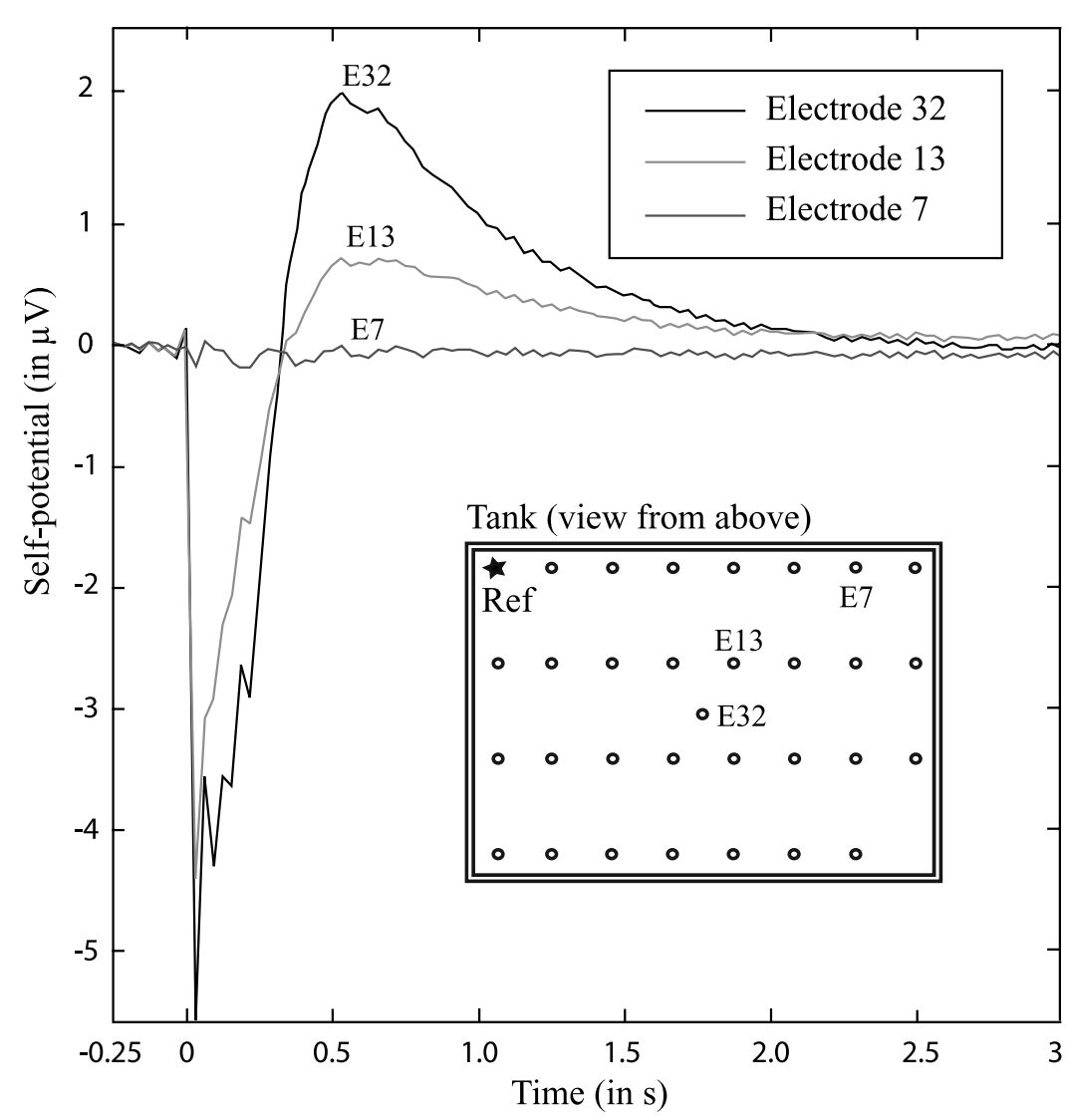

Figure 4. Electrograms for some selected electrodes (electrodes E32, E13, and E7) during the injection experiment (Experiment \#1). Electrode E32 is located just above the source while electrode E7 is the furthest. Note the change of polarity on the electrograms of electrodes E32 and E13 at time $t=0.35 \mathrm{~s}$.

(with respect to the reference electrode) centered above the pumping point (Figure 5). Consequently, injecting and pumping produce sign inverted self-potential anomalies. The maximum amplitude of the self-potential anomaly was $+5 \mu \mathrm{V}$ (Figure 6), which is a magnitude similar to the injection experiment. This implies that a relatively similar mechanism is at play during these two experiments. In this experiment, there was no reversal of the polarity of the anomaly over time (Figure 6). We observed a relaxation of the anomaly after it reached its maximum amplitude with a duration of few seconds. This experiment was performed three times and was reproducible.

\subsection{Additional Experiments}

[22] In order to test the distribution of the self-potential in a vertical plane passing by the capillary, we performed two additional experiments. Experiment \#3 is an infiltration test but with an initial shock smaller than in Experiment \#1 (Figures 7 and 8). For this experiment, we observed no reversal of the electrical potential anomaly during the experiment. This maybe indicates that the observed reversal of the self-potential signal is due to a dashpot behavior of the syringe. The distribution of the electrical potential through a vertical section of the tank shows clearly that the maximum of the amplitude of the anomaly is associated with the position of the inlet of the capillary.

[23] Experiment \#4 is a pumping test experiment but with an initial shock smaller than in Experiment \#2 (Figures 9 and 10). The distribution of the self-potential signals during this experiment shows very clearly that the maximum of the self-potential anomaly is initially associated with the outlet of the capillary (at a depth of $15 \mathrm{~cm}$ ). However, it seems that the maximum of the self-potential anomaly shifts upward over time after the initial phase.

\section{Forward Modeling \\ 4.1. Infiltration and Pumping Experiments}

[24] Inside the capillary, the water obeys to electroneutrality $\left(\bar{Q}_{V}=0\right)$ while the pore water of the sand is not neutral as discussed above $\left(\bar{Q}_{V}=0.77 \mathrm{C} \mathrm{m}^{-3}\right)$. Therefore during the injection or the pumping experiments, the water has to go through the interface between the capillary and the sand (see Figure 11). As this interface corresponds to a finite drop in the value of $\bar{Q}_{V}$, the dominant source term in equation (12) is:

$$
\Im_{3} \approx \nabla \bar{Q}_{V} \cdot \mathbf{U}
$$

An analytical solution of this problem is developed in Appendix A. A comparison between this solution and the measured values for Experiment \#1 is displayed on Figure 12a. A comparison with the measurements of Experiment \#1 taken at the time where the self-potential signals reached their minima is shown on Figure 12b.This 


\section{Self-potential (in $\mu \mathrm{V})$}

a.

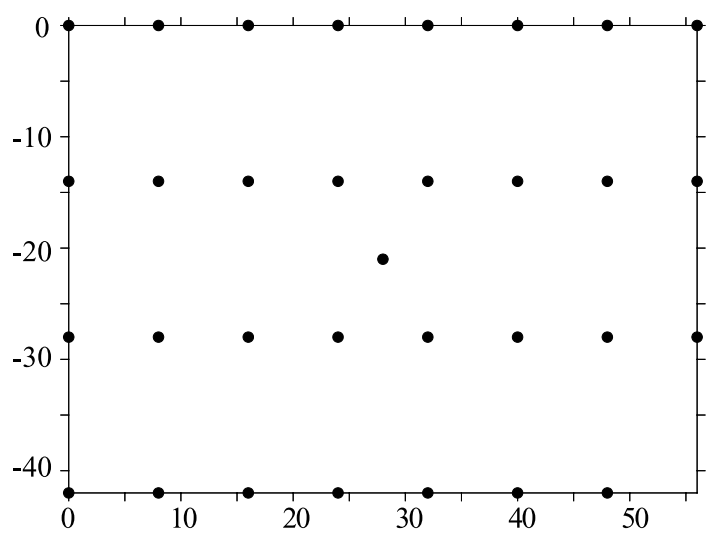

b.

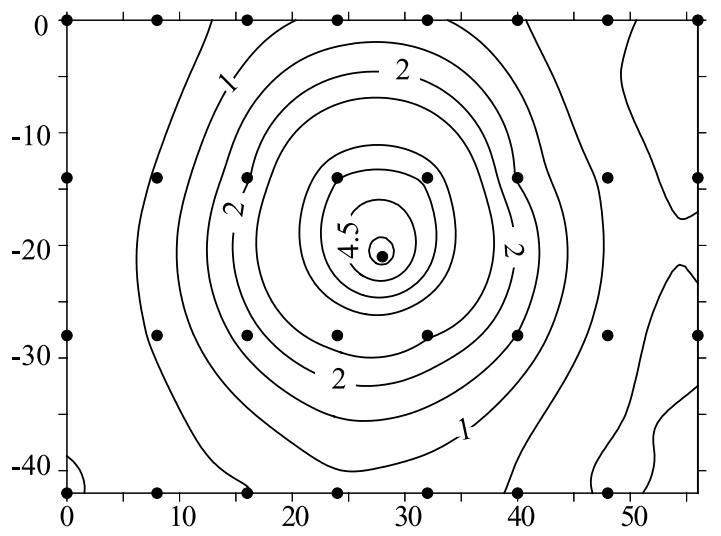

$\mathrm{t}_{1}=0.3 \mathrm{~s}$

c.

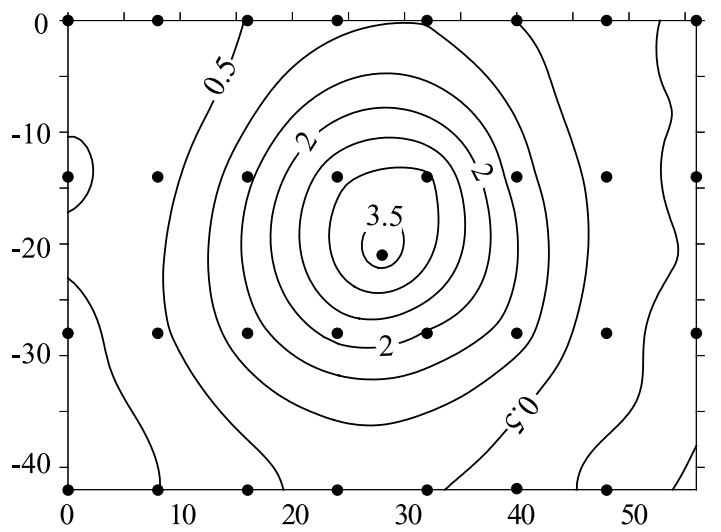

$\mathrm{t}_{2}=0.4 \mathrm{~s}$

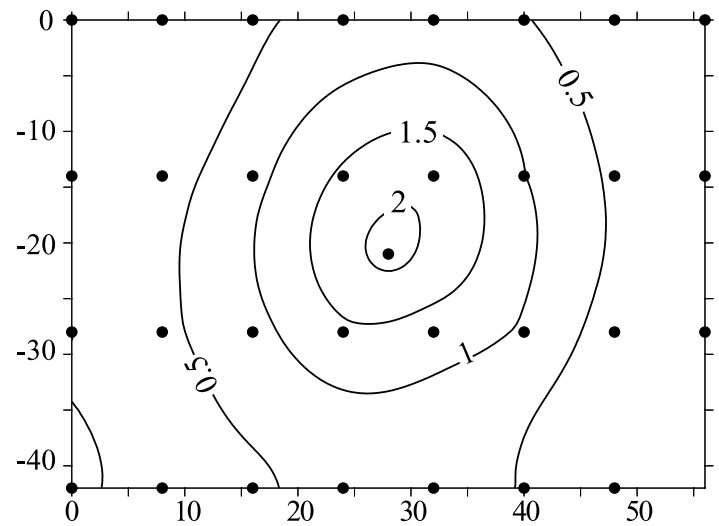

e. $\quad t_{3}=0.6 \mathrm{~s}$

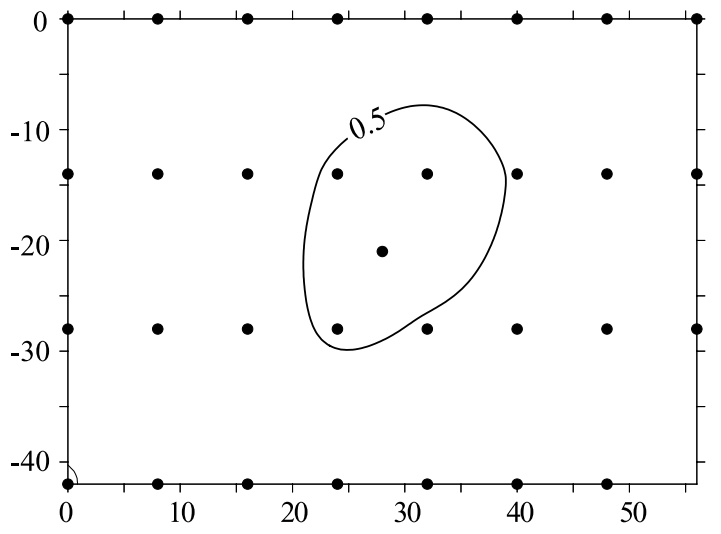

$\mathrm{t}_{4}=4.2 \mathrm{~s}$

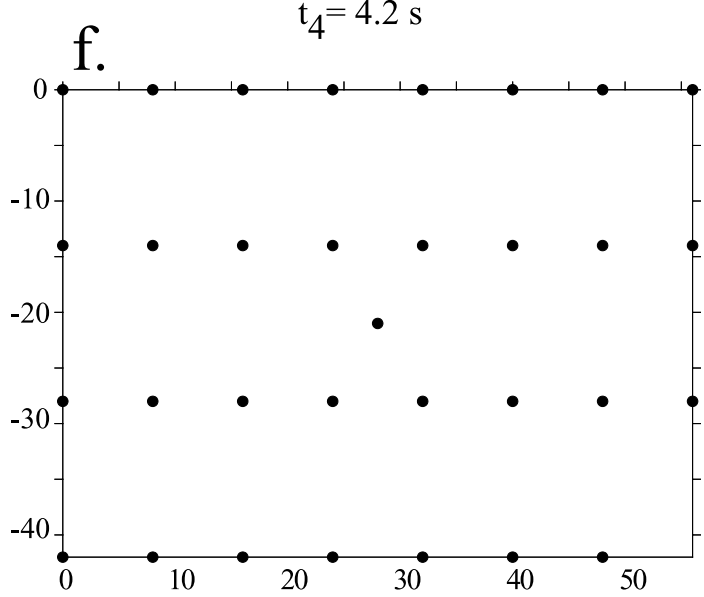

Figure 5. Time-lapse distribution of the self-potential measured at a depth of $3 \mathrm{~cm}$ below the ground surface $(t=0$ corresponds to the pulse pumping of water through the capillary, experiment \#2). At $t=$ $4.2 \mathrm{~s}$, the self-potential anomaly has vanished.

solution suggests that the source, in the initial stage of the experiment, was dipolar for Experiment \#1.

[25] The analytical solution developed in Appendix A requires a value of the excess fluid pressure at a given time. To model the evolution of the fluid pressure over time, we need to solve the hydrodynamic problem coupled with the poro-elastic equations and then to use the solution to solve the Poisson equation for the electrostatic potential. We use the commercial finite element code Comsol Multiphysics 3.3 to solve Darcy's law including deformation of the porous medium [e.g., Leake and Hsieh, 1997]:

$$
S_{\alpha} \frac{\partial \delta H}{\partial t}+\nabla \cdot(-K \nabla \delta H)=\xi \frac{\partial}{\partial t}(\nabla \cdot \mathbf{u})
$$




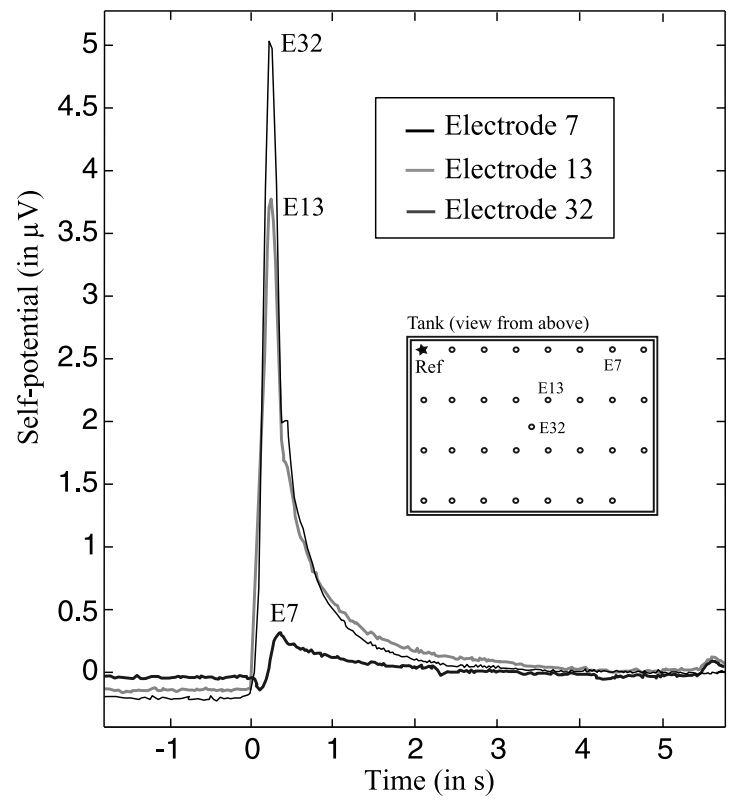

Figure 6. Electrograms for some selected electrodes (Electrodes E7, E13, and E32) located in the vicinity of the top surface of the tank during the pumping experiment (Experiment \#2). There is no change of the polarity during the course of the experiment.

where $\delta H=\delta p / \rho_{f} g$ is the change in hydraulic head (above or below the hydrostatic initial distribution $\left.H_{0}\right), S_{\alpha}=\rho_{f} g S$ is the poroelastic storage coefficient $\left(S_{\alpha}\right.$ is expressed in $\mathrm{m}^{-1}$ while $S$ is expressed in $\mathrm{Pa}^{-1}$ ), $K$ is the hydraulic conductivity (in $\mathrm{m} \mathrm{s}^{-1}$ ), $\mathbf{u}$ is the total displacement (in $\mathrm{m}$ ), $\xi$ is the Biot coefficient (dimensionless, see section 2$)$, and $\partial(\nabla \cdot \mathbf{u})$ is the time rate change in strain from the following equation for the displacement in plane strain for the solid grains [e.g., Leake and Hsieh, 1997].

[26] The equilibrium condition for the stress tensor is $\nabla \cdot \overline{\bar{\sigma}}=-\mathbf{F}$ where $\overline{\bar{\sigma}}$ is the stress tensor and $\mathbf{F}$ is the total body force plus the gradient of the pore fluid pressure. Indeed, the gradient of the pore fluid pressure acts as a force acting on the grains and responsible for the deformation of the medium. The total displacement in plane strain is given by solving [e.g., Leake and Hsieh, 1997],

$$
\frac{E}{2(1+v)} \nabla^{2} \mathbf{u}+\frac{E}{2(1+v)(1-2 v)} \nabla \cdot(\nabla \mathbf{u})=\xi \rho_{f} g \nabla \delta H,
$$

where $E$ is the Young modulus and $v$ is the Poisson's ratio. We use fixed head at the top surface of the tank and no-flow conditions on the other boundaries (n $K \nabla \delta H=0)$ including the surface of the tank and the surface of the capillary. The specific storage, the Young's modulus and the Poisson's ratio are not independent parameters. For incompressible solid grains and pores, the poroelastic storage coefficient is given by $S_{\alpha}=1 / K+\phi / K_{f}$ where $1 / K$ and $1 / K_{f}$ are the compressibility of the porous medium and the compressibility of the pore water $\left(K_{f}=3.3 \mathrm{GPa}\right)$, respectively. Using the relationship given by Wang [2000] (see his Table B1, Appendix B), we have $S_{\alpha}=3(1-2 v) / E+$ $\phi / K_{f}$. The initial condition for the fluid pressure was the hydrostatic distribution. The displacement of the medium was equal to zero on the walls of the tank. We adopted a free deformable surface at the top surface of the tank. Equations (14) and (15) are discretized using the finite element method and triangular meshing.

[27] The 2D numerical simulations were performed according to the following procedure. We first solved the hydromechanical problem (equations (14) and (15)) and then used the solution of the hydromechanical problem to determine the streaming current source density $\mathbf{J}_{\mathrm{S}}$. Finally, we solved the Poisson's equation for the streaming potential $\psi$. We used the values of the electrical conductivity and the voltage and current coupling coefficients reported in section 3.2. We assigned the boundary condition $\mathbf{n} \cdot \nabla \psi=$ 0 at all the insulating boundaries of the tank. The voltage reference was placed at the same location as the reference electrode in the laboratory experiment (see Figure 2). To perform the simulations, we used the following set of parameters: $E=6 \times 10^{7} \mathrm{~Pa}, v=0.4, S_{\alpha}=1.3 \times 10^{-8}$ $\mathrm{Pa}^{-1}, k=2.5 \times 10^{-12} \mathrm{~m}^{2}$, and $\xi=1$ for the sand (determined from the values reported in section 2 and the data published in the literature) and a dynamic viscosity for the pore water equal to $\eta_{f}=10^{-3} \mathrm{~Pa}$ s for water.

[28] The overpressure $\left(\delta p=p-p_{H}\right.$ where $p$ is the total fluid pressure and $p_{H}$ is the hydrostatic pressure) at the end of the capillary was estimated by trial and error to be $17 \mathrm{~Pa}$ $(1.2 \mathrm{~cm}$ of pressure head). These results show that the hydromechanical model discussed above is consistent with the measured self-potential distribution just after the pulse injection of water and over time.

[29] Using an excess fluid pressure of $17 \mathrm{~Pa}$ at the end of the capillary, we obtain the displacement and the fluid pressure distribution shown on Figure 13. The self-potential signal computed at electrode \#32 is shown on Figure 14. It shows clearly a change in polarity of the self-potential signal over time in agreement with the observed selfpotential signals (see Figure 4). This implies a change in the polarity of the excess pore fluid pressure (with respect to the hydrostatic pressure) inside the capillary. A profile of the self-potential signal at a depth of $3 \mathrm{~cm}$ is shown on Figure 15 at two characteristic times corresponding to the occurrence of the extrema of the development of the selfpotential anomaly.

[30] The influence of permeability upon the self-potential signals at electrode \#32 is shown on Figure 16. Clearly the permeability of the medium controls not only the intensity of the self-potential signal at time $t_{2}$ but also the relaxation of the self-potential anomaly over time after time $t_{2}$. This means that the time variation of the self-potential signals can be used to invert the hydraulic diffusivity and therefore the poro-elastic storage and the hydraulic conductivity of the medium.

\subsection{Pumping Experiment}

[31] In the pumping experiment (Experiment \#2), the drop in the charge density through the inlet of the capillary is responsible for a vertical dipole oriented upward. To test this assumption, a comparison between the analytical solu- 

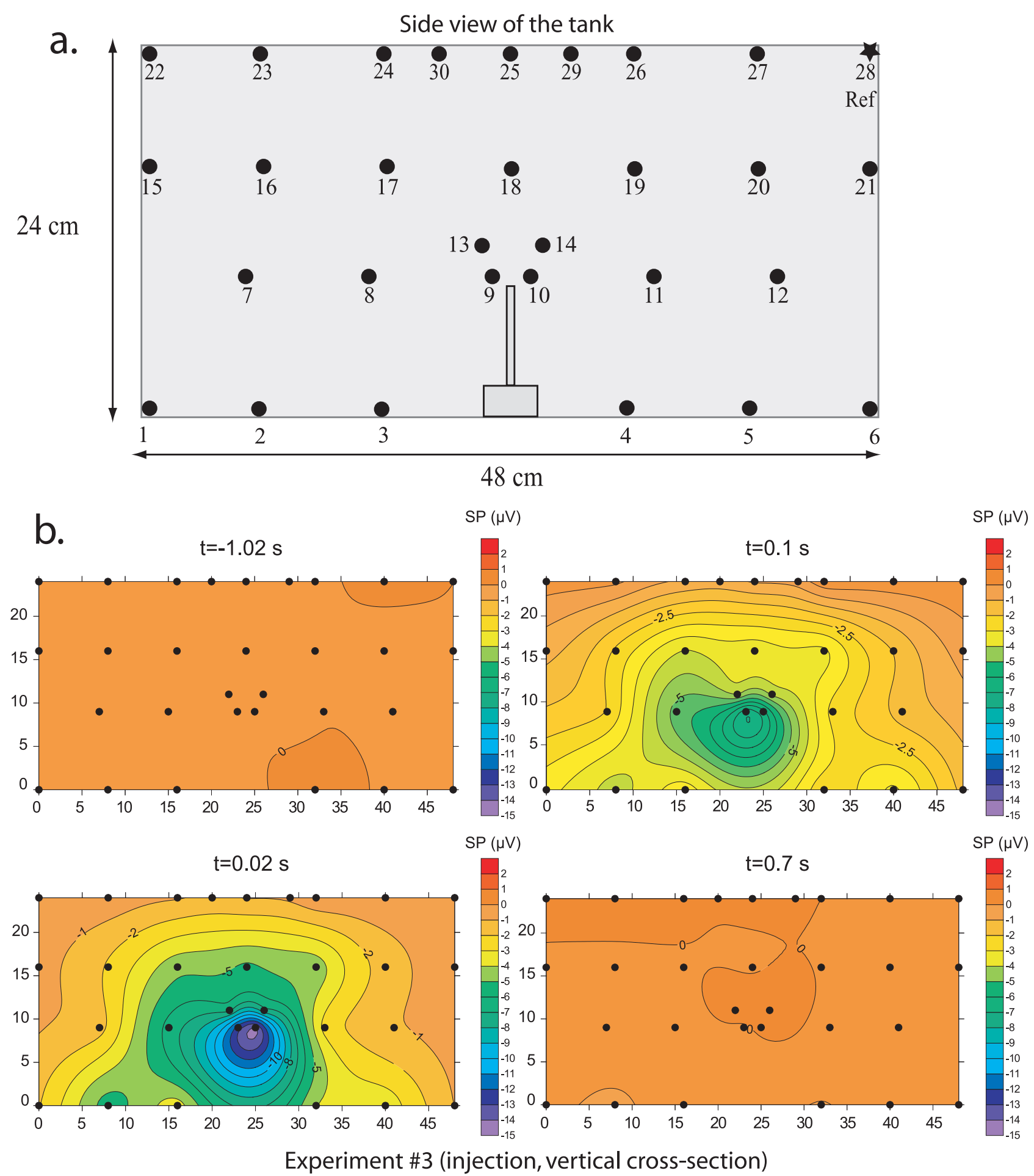

Figure 7. Injection experiment (Experiment \#3). (a) Side view of the tank with the position of the electrodes (\#28 is the reference) and the position of the capillary. (b) Snapshots of the electrical potential distribution of the self-potential at four different time-lapses. The shock created by the hammer stroke is smaller than in Experiment \#1.

tion developed in Appendix A and the measured potentials is given on Figure 12c. The measured self-potential data and those determined from the numerical model were found to be in fair agreement (see Figure 12d). Therefore the mechanism that yields to the development of the selfpotential anomalies is similar for both the injection and the pumping experiments. The change of the sign of the 


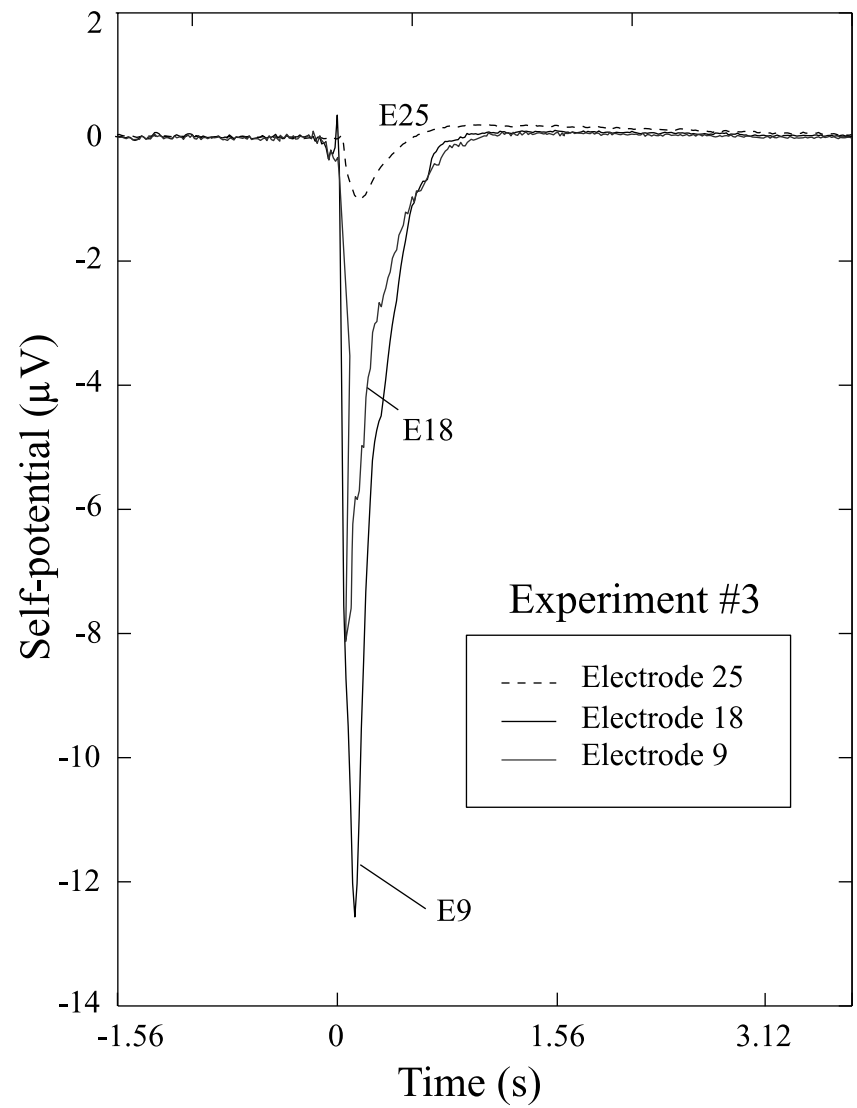

Figure 8. Injection experiment (Experiment \#3). Time variation of the self-potential of three selected electrodes.

anomaly between the two experiments is due to the direction of the flow of the water at the inlet/outlet of the capillary.

\section{Source Localization}

[32] Source localization of self-potential data is a relatively new and challenging field. Source localization can be performed using signal analysis algorithms (such as wavelet analysis or cross-correlation methods) or by minimization of a cost function using regularization (e.g., compactness applied to the inverted source) as shown recently by Minsley et al. [2007]. Minimization techniques can also be applied by specifying a simple source model and inverting the properties of this source model (such as depth of the source, direction of the dipolar moment, and intensity of the source, see Paul [1965], Rao and Babu [1984], Murty and Haricharan [1985]). In the analysis made below, we apply the wavelet technique to determine the properties of the source. This approach has the advantage that it does not require specifying the order of the multipole responsible for the self-potential signals recorded at the top surface of the tank. Once the order of the multipole has been determined, a cross-correlation technique can be used to infer the position of the source at depth.

\subsection{Use of Wavelet Analysis}

[33] We now consider one self-potential profile passing through the peak of the 2D self-potential anomaly. This self- potential profile is taken just after the injection or the pumping of water. Continuous wavelet analysis is used to locate the position of the source and its multipolar index [Grant and West, 1965; Holschneider, 1995; Fedi and Quarta, 1998; Mallat, 1999].

[34] In the initial stage of the deformation of the medium and for both injection and pumping experiments, the dominant source term in equation (12) is $\Im_{3} \approx \nabla \bar{Q}_{V}$. U. If the distance between the observation electrode and the source volume is much larger than the size of the source (here the diameter of the capillary), we can assume that the selfpotential field is created by a single homogeneous point source. This is the case here. In addition, we considered two-dimensional space to simplify the geometrical presentation and we assume that the electrical resistivity is constant in space, so that the equation (9) can be simplified to $\nabla^{2} \varphi=\Im_{3} / \sigma$. To show that these assumptions are compatible with the conditions of the experiment described in section 3, we first derive the source depth, and then estimate the multipolar index of the source.

[35] The idea of the method is to use a special type of wavelets called Poisson wavelets. These wavelets are related to the upward continuation filter $P a(x)$, transforming the potential field $\varphi(x)$ measured at a distance $Z$ to the values at the depth $Z+a$. Because of the scaling properties of wavelets and homogeneity property of the source, the wavelet coefficients $W(a, u)$ of potential fields at different scales $a$ exhibit a conelike structure converging to the location of the source, while the attenuation of the local maxima of the wavelet coefficients reveal the multipolar index of the source. In two-dimensional space, if the potential field $\psi(x)$ is excited by an homogeneous source $\Im$ located at depth $z_{0}$ and measured at the surface $(z=0)$, the corresponding wavelet coefficients in the upper half plane obey a power law, with the exponent $\beta=-\gamma+\alpha+1$ related to the order $\gamma$ of the chosen wavelets, the nature of the field and the multipolarity index $\alpha$ of the source.

[36] The depth $z_{0}$ can be obtained as the intersection point of the local maxima of the wavelet coefficients (wavelet ridges). Once the depth is known, the exponent $\beta$ can be determined as the slope of $\log \left(|W(a)| / a^{\gamma}\right)$ versus $\log \left(a+z_{0}\right)$.

[37] The Poisson wavelets are obtained with the help of the upward continuation filter [Grant and West, 1965],

$$
P_{a}(x)=\frac{1}{\pi} \frac{a}{a^{2}+x^{2}}
$$

Differentiating $P_{a}(x)$ with respect to $x$ and $a$ yields the corresponding first-order "horizontal" $g_{x}^{\gamma}(x)$ and "vertical" $g_{z}^{\gamma}(x)$ wavelets. Then the following $\gamma$-order derivative over $x$ gives the $\gamma$ order horizontal wavelets:

$$
\begin{gathered}
g_{x}^{\gamma}=\partial_{x}^{\gamma} P^{1}(x), \\
\hat{g}_{x}^{\gamma}(u)=(i 2 \pi|u|)^{\gamma} e^{-2 \pi|u|},
\end{gathered}
$$



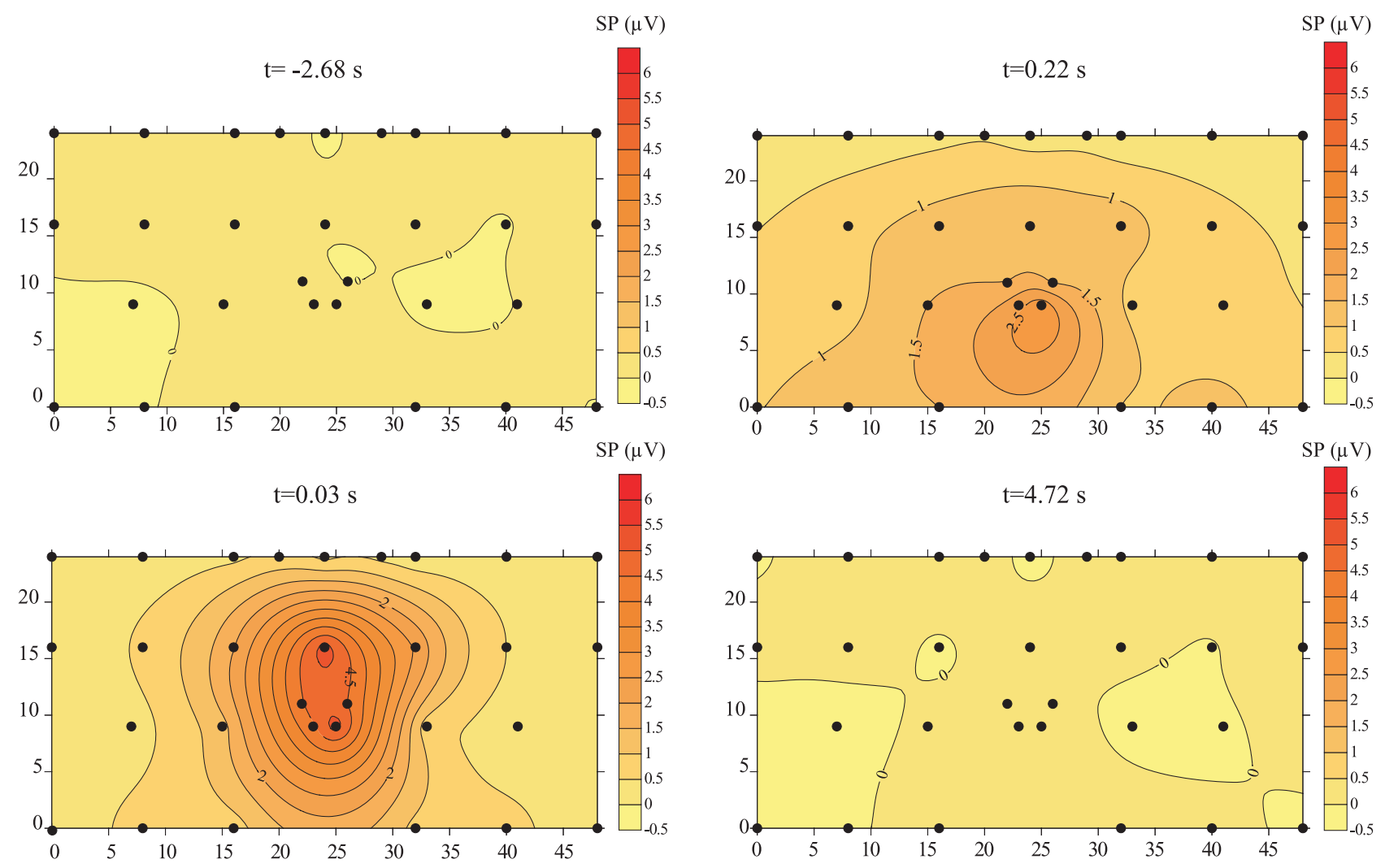

\section{Experiment \#4, Pumping, Vertical cross-section}

Figure 9. Pumping experiment (Experiment \#4). Snapshots of the electrical potential distribution of the self-potential at four different time-lapses. The shock created by the hammer stroke is smaller than in Experiment \#2.

where $\hat{g}_{x}^{\gamma}(u)$ is the Fourier transform of $g_{x}^{\gamma}$. Similarly the vertical wavelet of order $\gamma$ is,

$$
\begin{gathered}
g_{z}^{\gamma-1}=\left.\partial_{x}^{\gamma-1} \partial_{a} P_{a}(x)\right|_{a=1}, \\
\hat{g}_{z}^{\gamma}(u)=(i 2 \pi|u|)^{\gamma-1}(-2 \pi|u|) e^{-2 \pi|u|}, .
\end{gathered}
$$

The first order complex analytic wavelet $g_{c}^{\gamma}=g_{x}^{\gamma}+i g_{z}^{\gamma}$ (named the "Cauchy wavelet") is used to estimate also the orientation of the source. In Fourier domain, this wavelet is,

$$
\hat{g}_{c}^{\gamma}(u)=(-2 \pi|u|)^{\gamma} e^{-2 \pi|u|}
$$

In both sandbox experiments, the self potential anomaly along the diagonal attenuates almost totally at the sides of the sandbox. The self-potential profile is linearly interpolated to obtain two self-potential values per centimeter (113 points in total). The position $x=0$ corresponds to the center of the tank, above the position of the capillary. The self-potential data are zero-padded on both sides of the profile to fill a 1024 points vector (Figure 17a). Figure 17b shows the wavelet transform using a first order $(\gamma=1)$ vertical $g_{z}^{1}$-wavelet. In this case, equation (15) yields,

$$
W(u, a)=\left(\frac{a}{a^{\prime}}\right)\left(\frac{a^{\prime}+z_{0}}{a+z_{0}}\right)^{\alpha} W\left(u \frac{a^{\prime}+z_{0}}{a+z_{0}}, a^{\prime}\right)
$$

[38] Areas in dark grey scales in Figure 17b corresponds to low values of the wavelet coefficients, while clear grey scales in Figure $17 \mathrm{~b}$ show high values of the wavelet coefficients. The local maxima of the wavelet coefficients are shown by a dotted line. The cone-line structure of the wavelet coefficients converges to the source position $z_{0}=$ $12 \mathrm{~cm}$ below the position of the electrodes. This depth is consistent with the position of the inlet/outlet end of the capillary. The value of the coefficient $\beta$ is equal to slope of $\log \left(|W(a)| / a^{\gamma}\right)$ versus $\log \left(a+z_{0}\right)$. We find $\beta=\alpha=-2.2$ (see Figure 17c). This value corresponds approximately to a dipole source $(\beta=-2$ for a perfect dipole). Finally, the phase iso-value of the complex wavelet transform $g_{c}^{1}$, at the source position, gives the directivity of the polarization vector from the vertical direction. This angle is about $1^{\circ}$ (at the position $x=0$ ), which means that the dipole is nearly vertical.

[39] Analysis of the self-potential data related to the pumping experiment (see Figure 18) yields a source depth equal to $z_{0}=13 \mathrm{~cm}$ (the depth of the inlet of the capillary is $12 \mathrm{~cm}$ ) and we find $\beta=\alpha=-2.3$ (the source is nearly dipolar in nature) (see Figure 18c). From the wavelet analysis, the source appears to be a vertical dipole pointing upward, in agreement with the model developed in section 4 . 


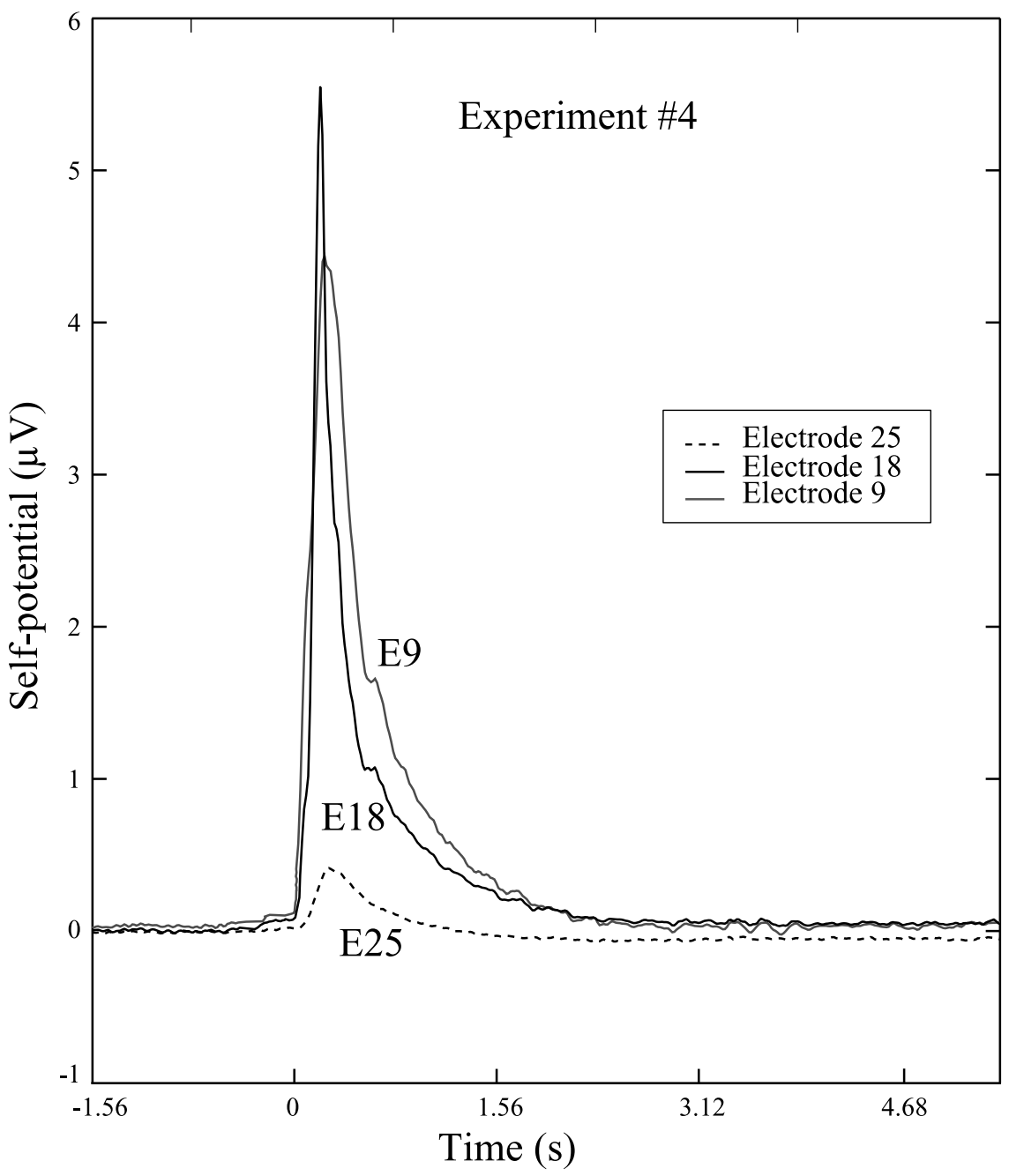

Figure 10. Pumping experiment (Experiment \#4). Time variation of the self-potential of three selected electrodes.

\subsection{Semblance Function}

[40] The wavelet analysis presented in Figures 17 and 18 cannot accommodate information concerning the topography and the electrical resistivity distribution of the conductive medium. Therefore for the purpose of elaborating a method that could be applied to an active volcano, we tested another method based on the cross-correlation between the Green function derived from the linear operator associated with Poisson equation and the measured self-potential anomaly divided by its power. This approach has been used recently by Revil et al. [2003] to determine the geometry of the water table of unconfined aquifers. We used the 3D generalization of the cross-correlation density algorithm developed by Revil et al. [2001] to locate a dipolar source inside the medium [see Iuliano et al., 2002]. We considered a cartesian coordinate system $(x, y, z)$ with the $z$ axis positive downward. We assumed that the electrical field $\mathbf{E}(\mathbf{r})$ was due a single dipole with a moment $\mathbf{d}$,

$$
\mathbf{E}(\mathbf{r})=\mathbf{d} \nabla \mathbf{G},
$$

where $\mathbf{G}$ is the Green function of the source assumed to be dipolar. $\mathbf{G}$ can be obtained using the derivation of the source function described in Appendix A. We note $E$ the modulus of the electrical field $\mathbf{E}(\mathbf{r})$. The power associated with the electrical field is,

$$
\begin{gathered}
\wp(E)=\int_{S} E^{2}(\mathbf{r}) d S, \\
\wp(E)=\sum_{v} d_{v} \int_{S} \mathbf{E}(\mathbf{r}) \frac{\partial \mathbf{G}}{\partial v_{P}} d S,
\end{gathered}
$$

where $v=x, y, z, v_{p}=x_{p}, y_{p}, z_{p}$. The projection of $S$ onto the $(x, y)$ horizontal plane is adapted to a rectangle with sides of total length $2 X$ and $2 Y$ along the $x$ - and $y$ axis, respectively, and corresponding to the horizontal dimensions of the tank. We note $g(z)$ the topography regularization factor defined by,

$$
g(z)=\left[1+\left(\frac{\partial z}{\partial x}\right)^{2}+\left(\frac{\partial z}{\partial y}\right)^{2}\right]^{1 / 2}
$$




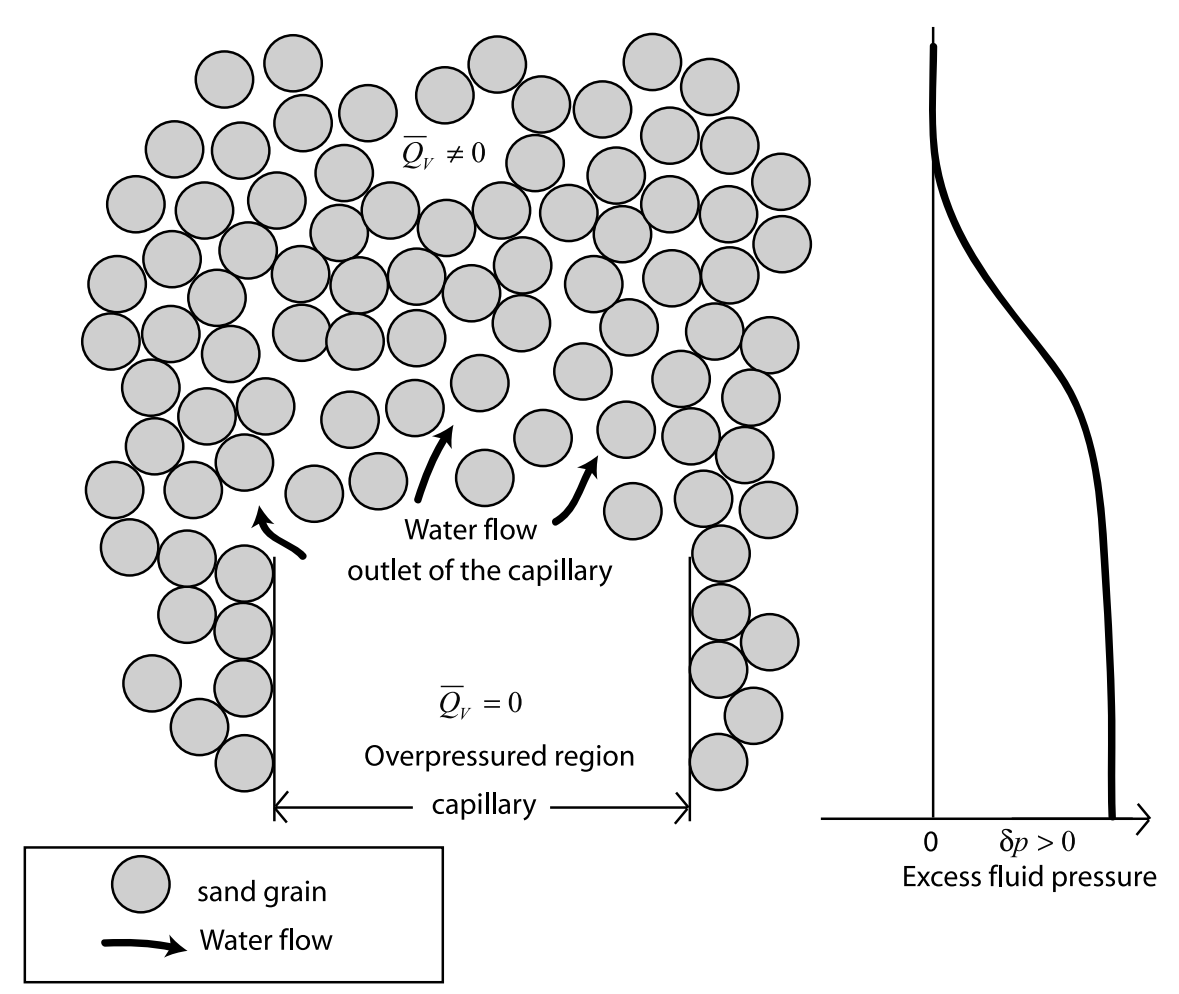

Figure 11. The boundary between the inlet / outlet of the capillary and the surrounding sand is characterized by a drop in the charge density per unit pore volume $\bar{Q}_{V}$. The resulting dipolar momentum vector $\mathbf{P}$ is oriented downward. Its position is likely to be located slightly above the capillary outlet. Its strength is expected to decrease over time because of the decrease of the fluid pressure over time due to the diffusion of the pressure field around the outlet of the capillary.

which in the present case is $g(z)=1$ (no topography). Iuliano et al. [2002] introduced the following crosscorrelation product,

$$
\eta_{v}\left(r_{p}\right)=C_{v}^{p} \int_{-X}^{X} \int_{-Y}^{Y} \mathbf{E}(\mathbf{r}) \frac{\partial \mathbf{G}\left(\mathbf{r}_{p}-\mathbf{r}\right)}{\partial v_{p}} g(z) d x d y .
$$

The normalization constant $C_{v}^{p}$ is defined by,

$$
C_{v}^{p}=\left[\int_{-X}^{X} \int_{-Y}^{Y} E^{2}(\mathbf{r}) g(z) d x d y \int_{-X}^{X} \int_{-Y}^{Y} \frac{\partial \mathbf{G}\left(\mathbf{r}_{p}-\mathbf{r}\right)}{\partial v_{p}} g(z) d x d y\right]^{-1 / 2} .
$$

The semblance function is therefore the normalized scalar product between the form-anomaly factor indicated by the self-potential measurements at the ground surface and the form-factor associated with a hypothetical dipolar source located in the source volume. These cross-correlation densities have the following property,

$$
-1 \leq \eta_{v}\left(r_{p}\right) \leq 1 .
$$

The norm of the cross-correlation vector $\boldsymbol{\eta}\left(\eta_{x}, \eta_{y}, \eta_{z}\right)$ is given by,

$$
\eta\left(r_{p}\right)=\sqrt{\eta_{x}\left(r_{p}\right)^{2}+\eta_{y}\left(r_{p}\right)^{2}+\eta_{z}\left(r_{p}\right)^{2}}
$$

If the distribution of the electrical resistivity is heterogeneous (or possibly anisotropic), the Green function $\mathbf{G}$ should be computed at the locations of each electrode using a numerical method assessing the self-potential response obtained by putting elementary dipoles along the three Cartesian directions for each elementary cell of the ground. Then, it is possible to use the cross-correlation algorithm as above to determine the distribution of the cross-correlation densities (and its associated probability density) in the scanned subsoil.

[41] For the canonical problem of finding a dipole in a homogeneous ground, the position of the source is given by $\operatorname{Max}\left[\eta\left(r_{p}\right)\right]_{V}$ (where $\operatorname{Max}[f(x)]_{x 1}^{x_{1}}$ is the maximum of the function $f(x)$ over the support $\left.\left(x_{1}, x_{2}\right)\right)$. In other words, the position of the source is obtained by maximizing the semblance between the form-anomaly factor and the formfactor associated with the dipolar source distribution. The coordinates of the vector $\boldsymbol{\eta}\left(\eta_{x}, \eta_{y}, \eta_{z}\right)$ at this point provides the direction of the dipole moment $\mathbf{d}$ at the source. The cross-correlation density of finding a dipole responsible for the observed self-potential anomaly is shown on Figure 19 for the injection experiment and on Figure 20 for the pumping experiment. In the first case, the maximum of the cross-correlation density is located at position $S(0 \pm 2 \mathrm{~cm}$, $0 \pm 2 \mathrm{~cm}, 14 \pm 2 \mathrm{~cm}$ ) (so at a depth of $11 \pm 2 \mathrm{~cm}$ below electrode \#32). This is consistent with the actual position of the outlet of water in the tank, which is located at position $S(0 \mathrm{~cm}, 0 \mathrm{~cm}, 15 \mathrm{~cm})$. As explained in section 3 , it is not 


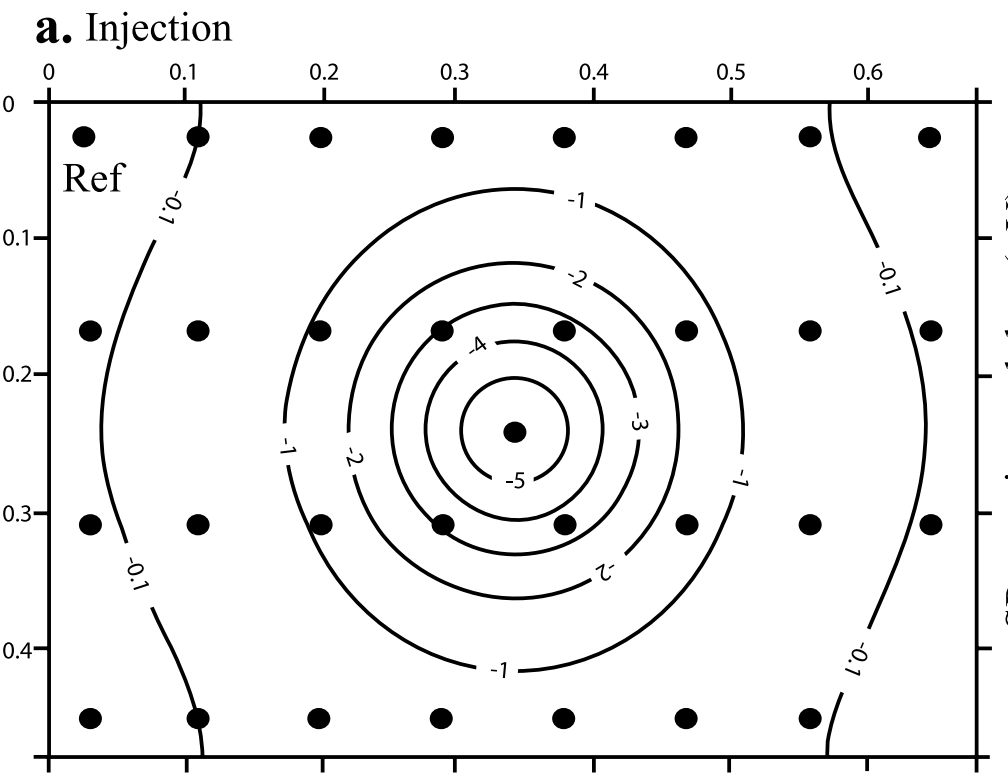

c. Pumping

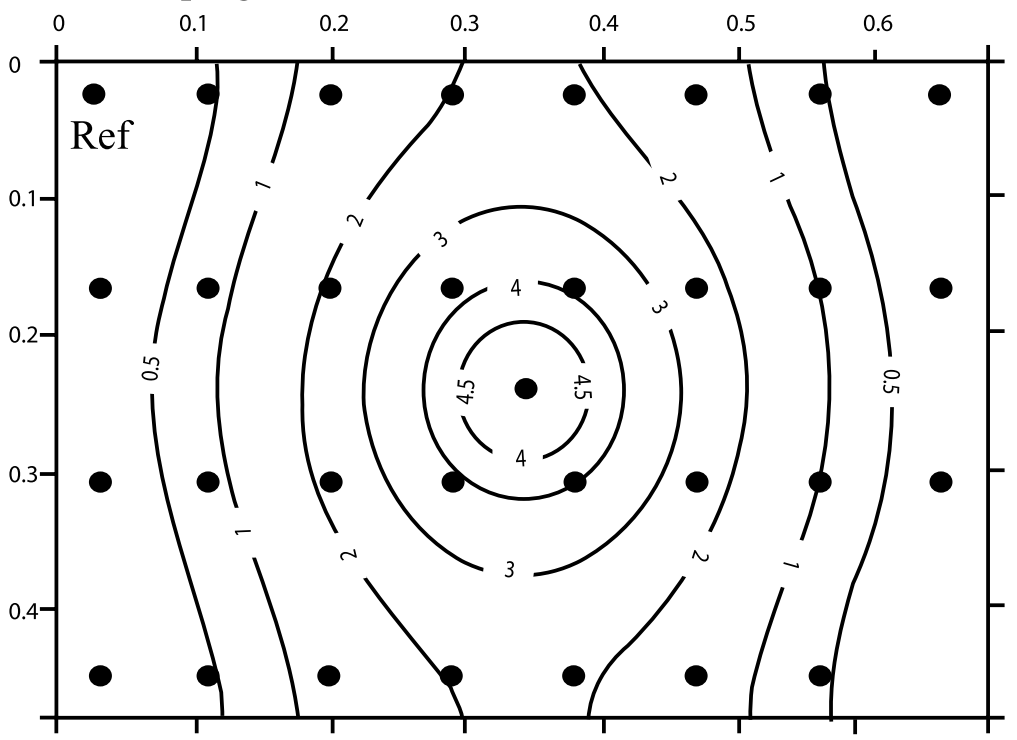

b.

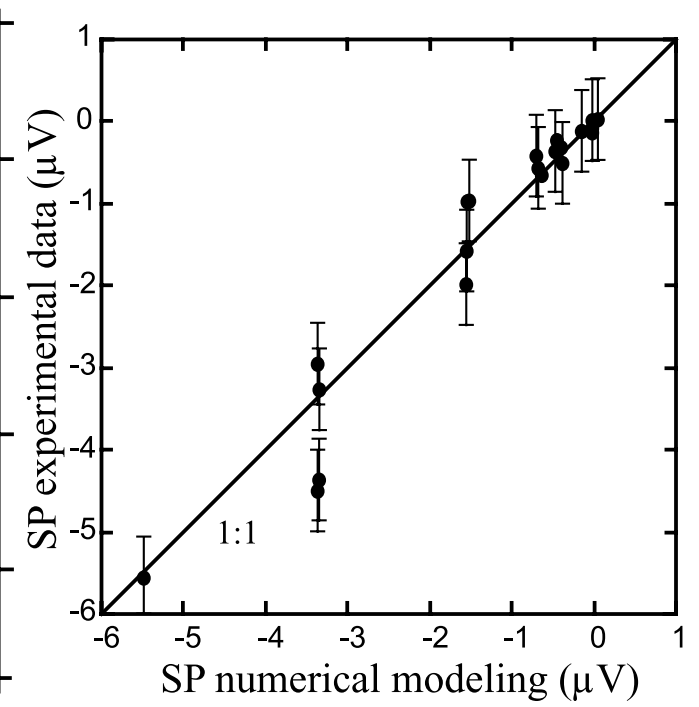

d.

Figure 12. Comparison between the analytical solutions for the self-potential response developed in Appendix A and the measured self-potential distributions. (a) Self-potential map, at a depth of $3 \mathrm{~cm}$ in the tank, for the infiltration experiment. The filled circles correspond to the position of the electrodes in the real sandbox experiment. (b) Comparison between the analytical solution and the measured self-potential signals at the top surface of the tank (at the peak of the intensity of the self-potential signals (Experiment $\# 1$ ). (c) Self-potential map for the pumping experiment from the analytical model. (d) Comparison between the analytical solution and the measured data for the pumping test (Experiment \#2).

surprising to localize the source slightly above the outlet of the capillary.

[42] In the case of the pumping test (Figure 20), the maximum of the probability density is located at position $S(0 \pm 2 \mathrm{~cm}, 0 \pm 2 \mathrm{~cm}, 16 \pm 1 \mathrm{~cm})$ (so at a depth of $13 \pm 2 \mathrm{~cm}$ below electrode \#32 in the second experiment). Accounting for the uncertainty given above, this is also consistent with the actual position of the inlet of water in the tank $S(0 \mathrm{~cm}$, $0 \mathrm{~cm}, 15 \mathrm{~cm})$. Therefore the cross-correlation algorithm is capable of identifying the position of the source responsible for the self-potential anomaly with a reasonable accuracy.

\section{Discussion}

[43] In this section, we discuss the level of sensitivity required to locate transient self-potential signals associated with fracturing in an active volcano. We consider a vertical fracture located at a depth of $H=1 \mathrm{~km}$ with a cross-section area of $S=100 \mathrm{~m}^{2}$. In volcanoes, a typical value of the 


\section{a. Time $\mathrm{t}_{1}$}

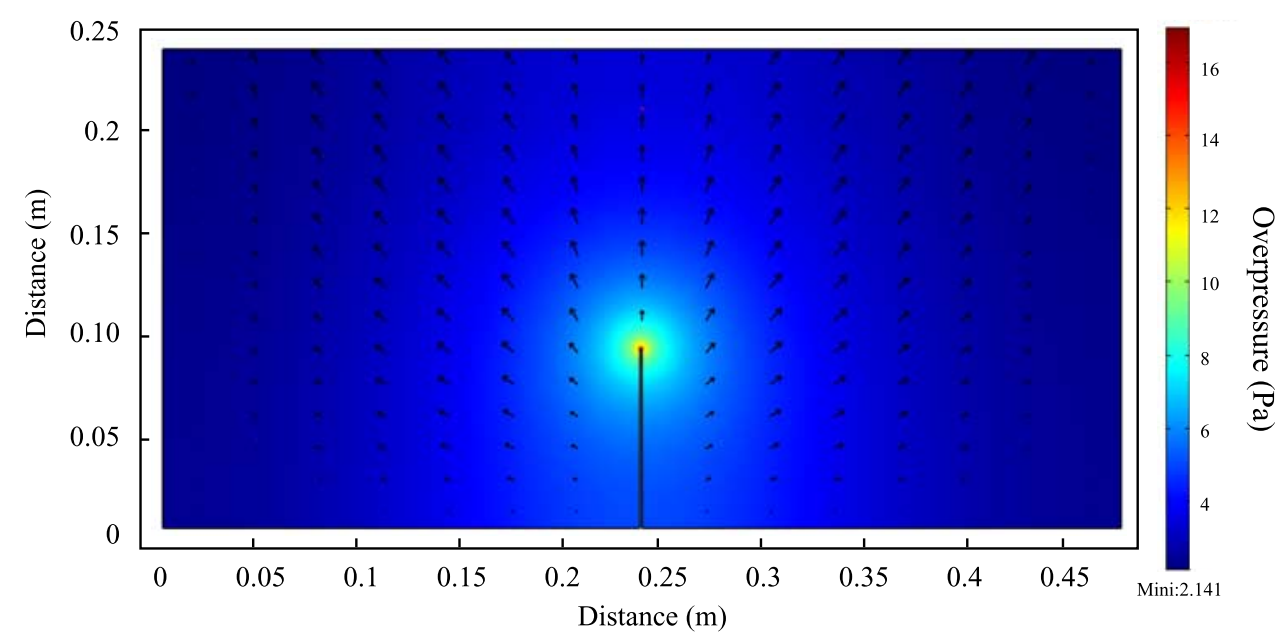

b. Time $t_{2}$

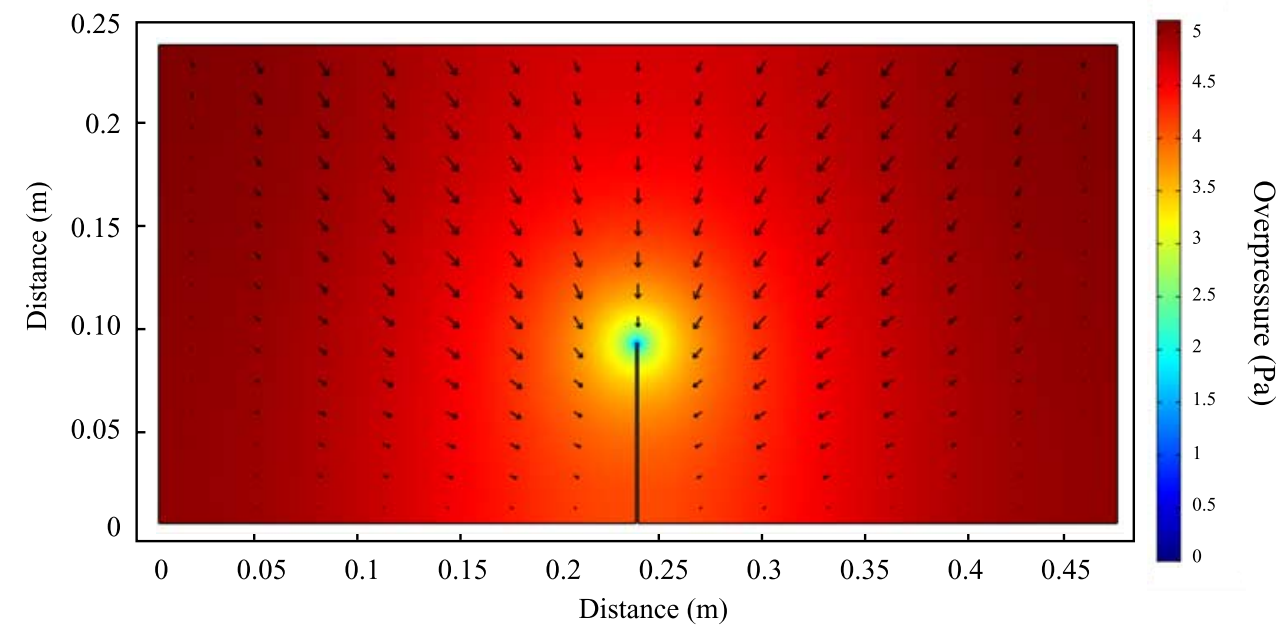

Figure 13. The arrows show the direction of the total displacement $\mathbf{u}$ (in $\mathrm{m}$ ) at time $t_{1}$ (a) and at time $t_{2}$ (b) respectively (see Figure 14). The colors code the fluid pressure above hydrostatic (in Pa). The change of polarity is due the reversal of the fluid flow due to the boundary condition expressed in term of fluid pressure at the end of the capillary. This polarity change is entirely due to the piston-like effect of the syringe.

voltage coupling coefficient $C$ is $-10 \mathrm{mV} \mathrm{m}^{-1}$ [e.g., Revil et al., 2003, 2004, and references therein]. The overpressure associated with fracturing is taken equal to the difference between the lithostatic stress and the hydrostatic stress. According to the analysis made in Appendix A, the maximum of the self-potential anomaly is given by $\varphi_{0} \approx(2 S / \pi H) C$ (with $C$ expressed in $\mathrm{mV} \mathrm{m}^{-1}$ ). With the parameters given above, this yields a self-potential transient signal of $\sim 1 \mathrm{mV}$. This value is largely above the threshold sensitivity of electrical sensors (probably $1 \mu \mathrm{V}$ once telluric and anthropic signals have been filtered out). For example in electroseismic investigations or for electromagnetic surveys, after filtering telluric currents and anthropic sources of noises, the sensitivity of measuring self-potential transient signals was found to be on the order of $1 \mu \mathrm{V}(1 \mathrm{nV}$ at the seafloor, Alumbaugh, D. L, personal communication, 2006). If the source is located at a depth of $100 \mathrm{~m}$, the self-potential transient anomaly recorded at the ground surface amounts to $\sim 10 \mathrm{mV}$, a value that can be easily monitored using a sensitive voltmeter and appropriate correction of the telluric currents in the frequency domain.

[44] In addition, electrical disturbances associated with fracturing would occur in the band of few Hertz. Other selfpotential sources like those associated with migration of water in the vadose zone and aquifers should occur at much lower frequencies [e.g., Linde et al., 2007]. In conclusion, it is likely that fracturing located at depths of a few hundreds meters to a few kilometers can be recorded by passive selfpotential measurements. The determination of the properties of the source (direction and location) requires the knowledge of the distribution of the electrical resistivity of the volcano. This distribution can be obtained though electromagnetic data or DC-electrical resistance tomography [e.g., Finizola et al., 2004; Finizola et al., 2006].

[45] One may also worries about the frequency dependence of the source. Because we are in the quasi-static regime of the Maxwell equations, the frequency dependence of the problems depends on the frequency dependence of 


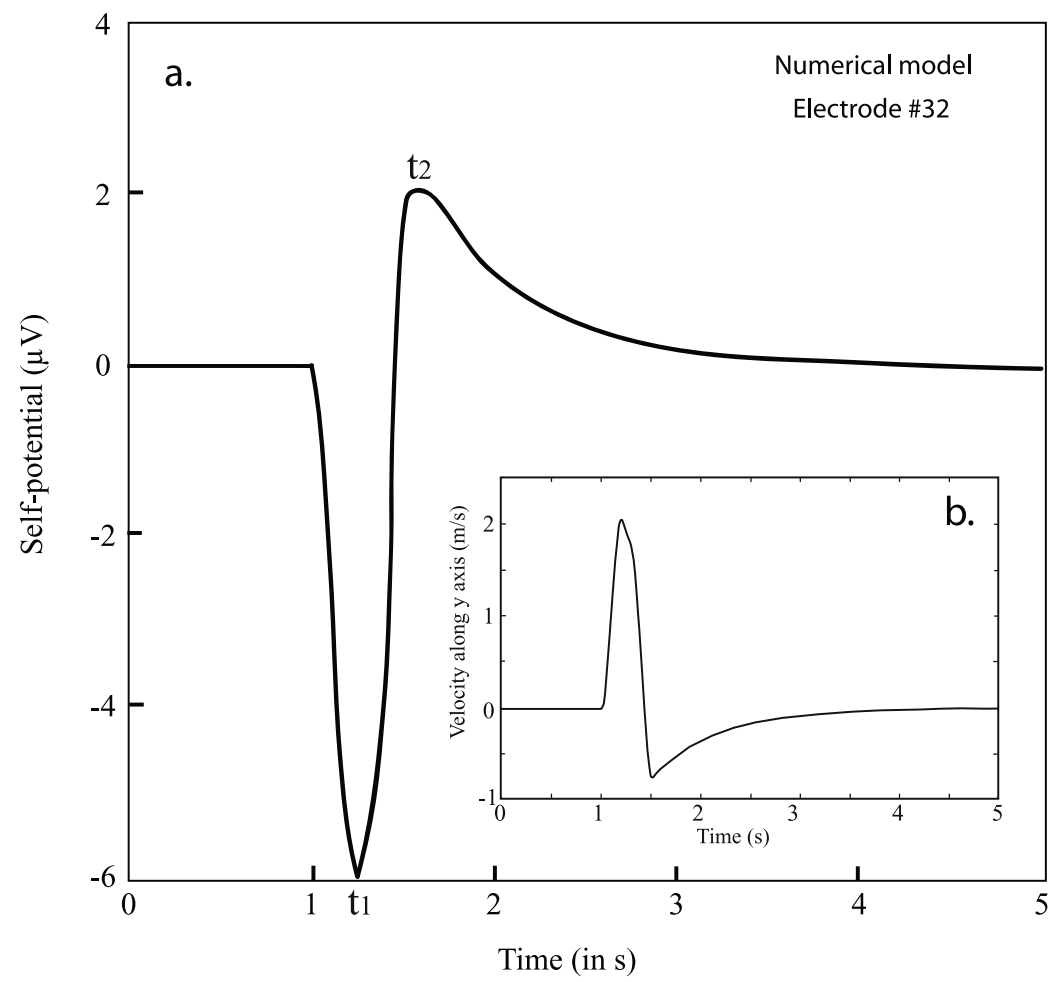

Figure 14. 2D numerical modeling of the self-potential response at electrode \#32 (Experiment \#1). (a) Note that the shape of the self-potential curve is similar to the measured self-potential signals (see Figure 4). (b) Computation of the fluid velocity at the end of the capillary. The reverse of the fluid velocity of the pore water is due to the fact that the fluid pressure is assigned equal to zero at $t=1.5 \mathrm{~s}$.

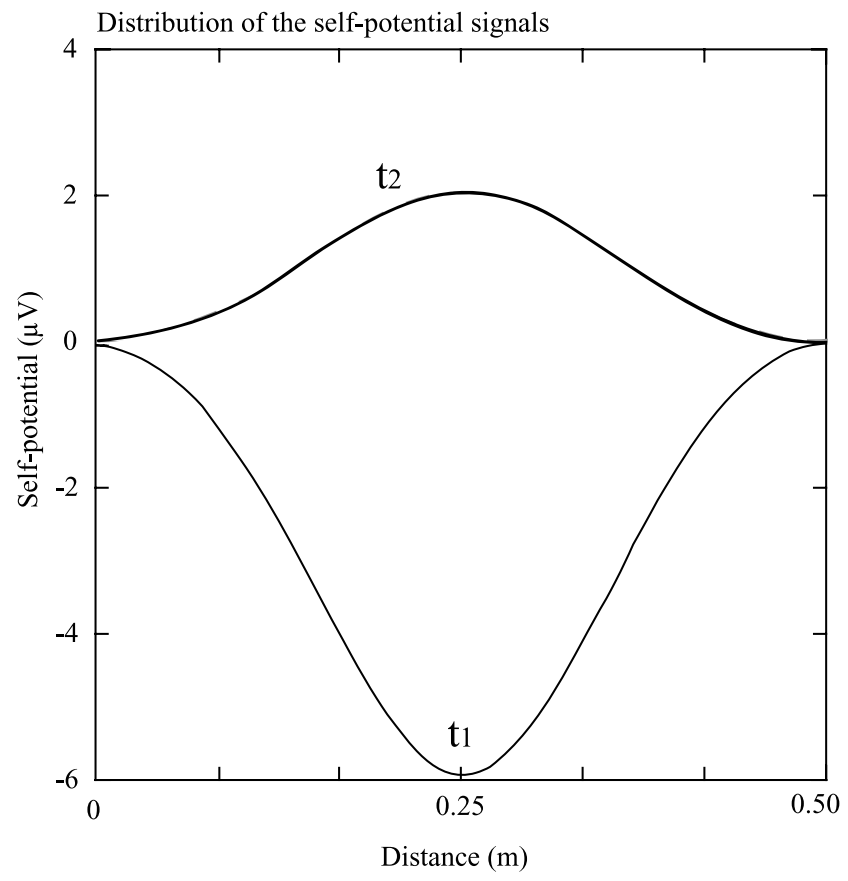

Figure 15. Distribution of the self-potential response at time $t_{1}$ and $t_{2}$ (see Figure 14) at a depth of $3 \mathrm{~cm}$ for the injection experiment. the electrical resistivity and the streaming potential coupling coefficient. At frequencies lower than $1 \mathrm{kHz}$, the frequency dependence of the electrical resistivity and the frequency dependence of the streaming potential coefficient are very small and can be safely neglected (see Olhoeft [1985] and Block and Harris [2006], respectively).

[46] Promising domains where this technique could also be applied are the monitoring of $\mathrm{CO}_{2}$ sequestration operations [e.g., Moore et al., 2004], dams and embankments [Gex,

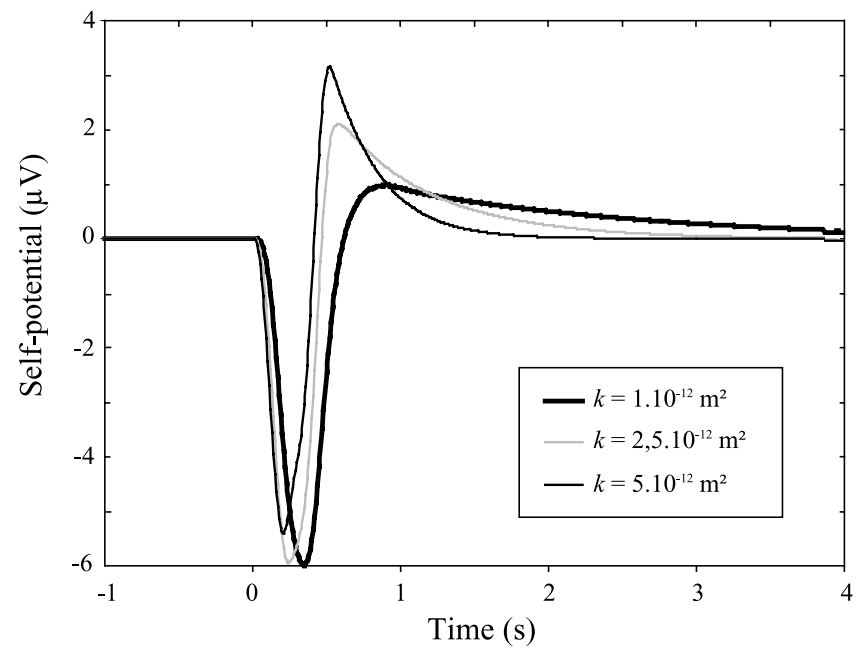

Figure 16. Simulated self-potential response at electrode $\# 32$ for different values of the permeability $k$ (Experiment $\# 1$ ). 

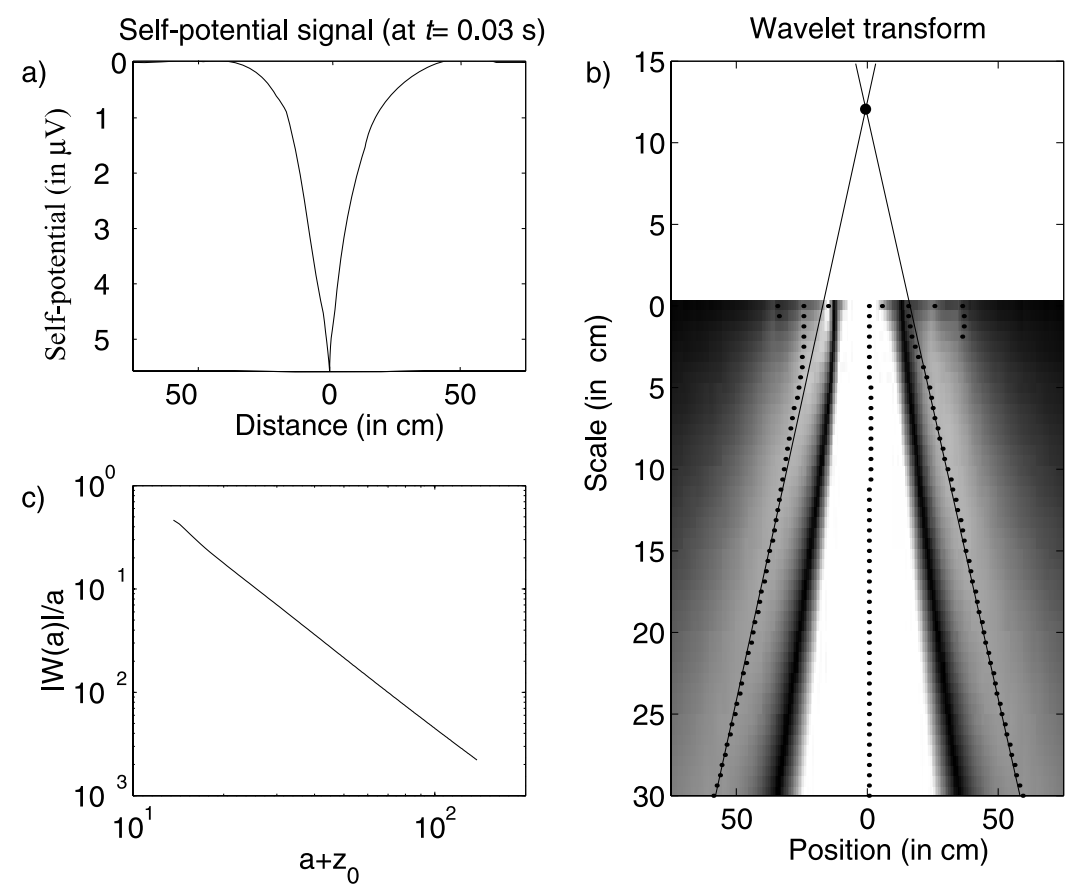

Figure 17. Wavelet analysis of the self-potential data at the maximum development of the self-potential anomaly in the case of the pulse injection of water. (a) Measured self-potential signals in $\mu \mathrm{V}$. (b) Continuous wavelet transform using a first order vertical wavelet. The solid lines show the wavelet coefficient maxima that intersect at the position of the source. (c) The order of the source is estimated from the slope $|W(a)| / a$ versus $a+z_{0}$ (the slope is equal to 2.2). It follows that the source corresponds to an electrical dipole.

Self-potential signal (at $t=0.03 \mathrm{~s})$

a)

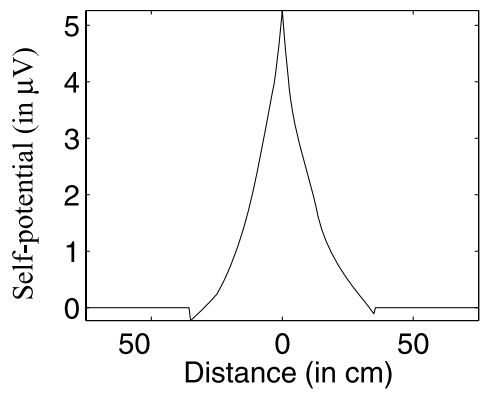

c)

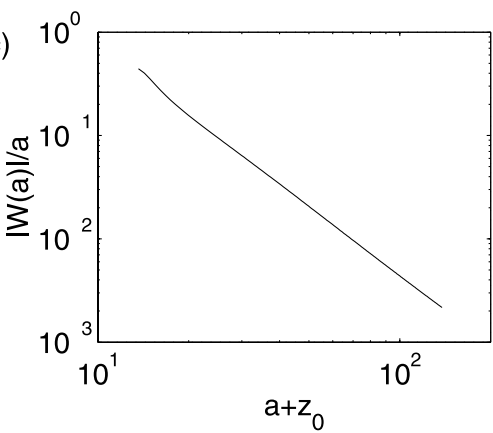

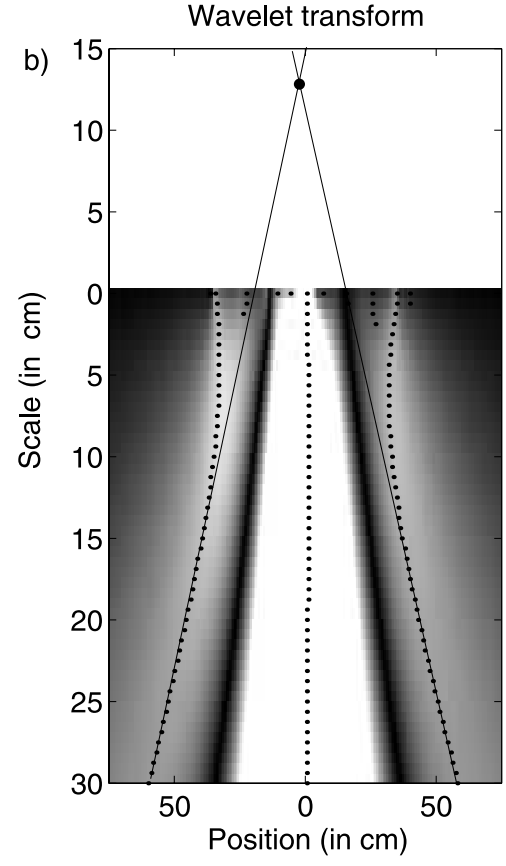

Figure 18. Wavelet analysis of the self-potential data at the maximum development of the self-potential anomaly in the case of the pulse pumping of water. (a) Measured self-potential signals in $\mu \mathrm{V}$. (b) Continuous wavelet transform using a first order vertical wavelet. The solid lines show the wavelet coefficient maxima that intersect at the position of the source. (c) The order of the source is estimated from the slope of $|W(a)| / a$ versus $a+z_{0}$. The source corresponds to an electrical dipole. 


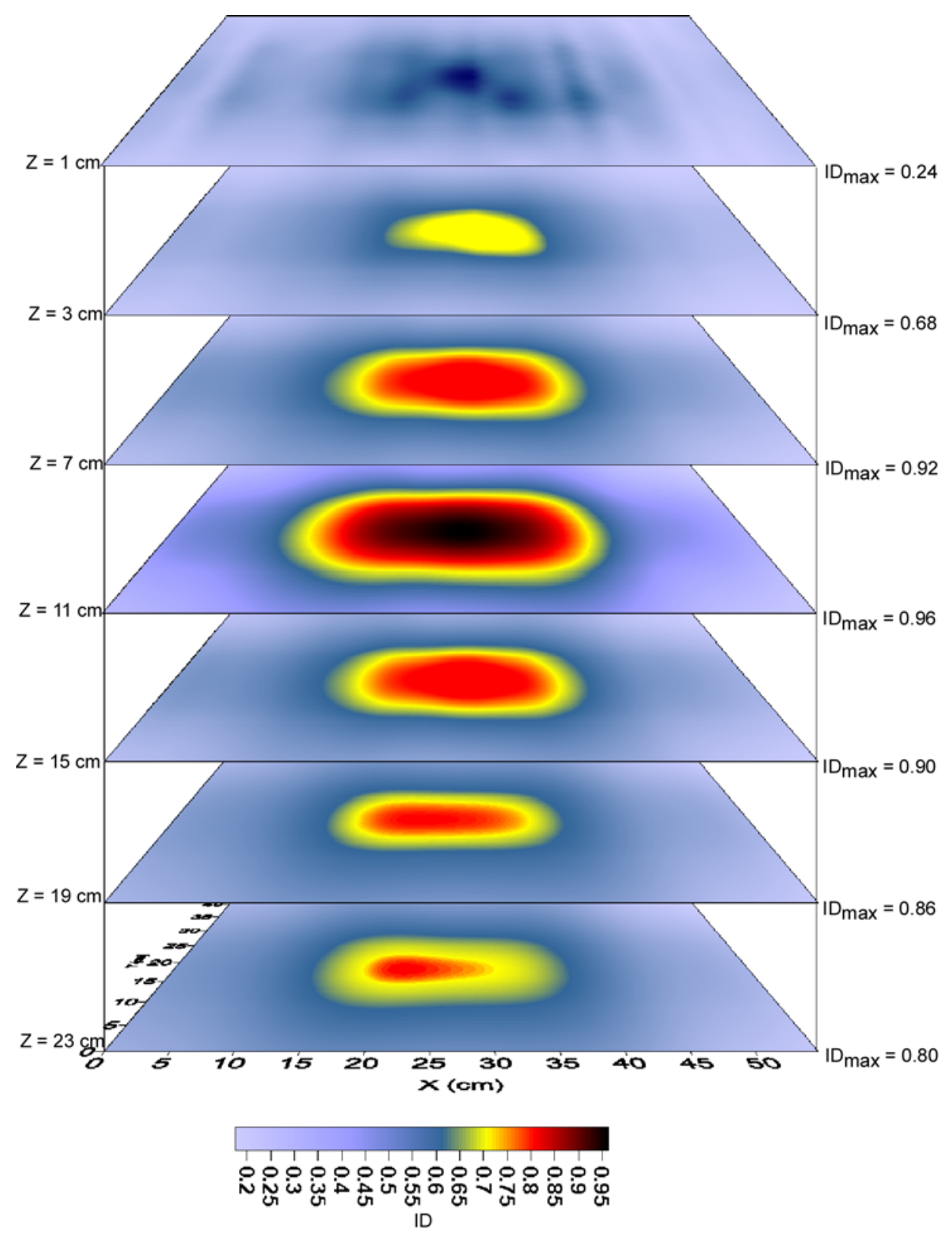

Figure 19. Distribution of the cross-correlation density over the tank (experiment \#1). The maximum of the cross-correlation distribution is located at a depth of $14 \mathrm{~cm}$ below the top surface of the tank. The numbers on the right side of the tomogram represent the maxima of the cross-correlation function for each horizontal plane. 


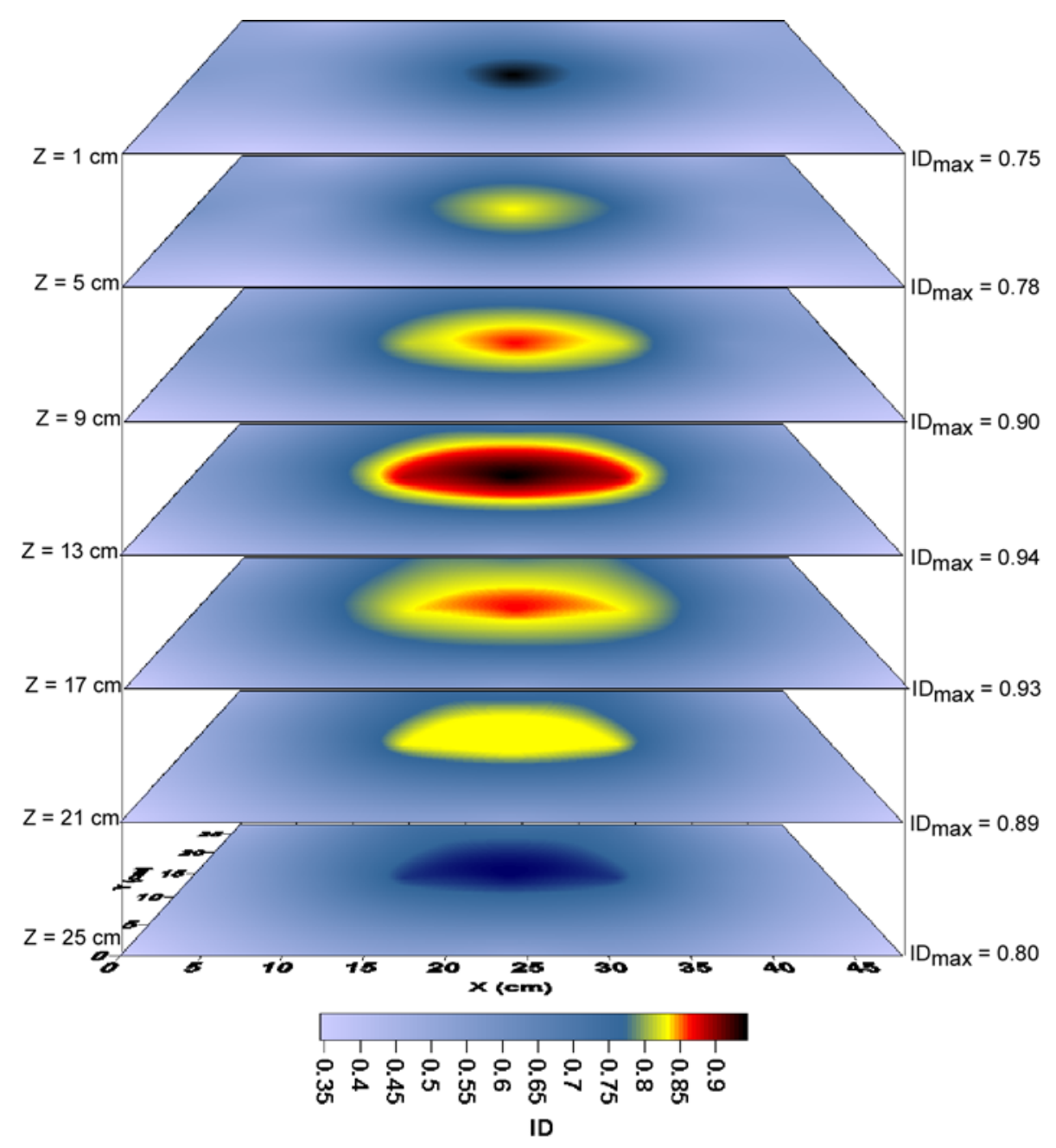

Figure 20. Distribution of the cross-correlation density over the tank for the pumping test (experiment \#2). The maximum of the cross-correlation distribution is located at a depth of $16 \mathrm{~cm}$ below the top surface of the tank. The numbers on the right side of the tomogram represent the maxima of the cross-correlation function for each horizontal plane.

1980; Merkler et al., 1989; Wilt and Corwin, 1989; Butler et al., 1990; Triumf and Thunehed, 1996; Sheffer and Howie, 2003; Bolève et al., 2007], glaciers [Kulessa et al., 2003], and the activity of faults [Park et al., 2007]. All hydromechanical Earth processes occurring in the conductive ground can be monitored by this method if their signal-to-noise ratio is high enough.

\section{Concluding Statements}

[47] We show that electric signals of electrokinetic nature are generated in response to hydromechanical disturbances in a water-filled electro-poro-elastic medium like a water saturated sand pack. To understand the underlying physics of this process, we performed four sandbox experiments. Prior to the experiments, the sandbox was filled with a wellsorted sand and infiltrated with an electrolyte of known composition and concentration. During the experiments, a known volume of electrolyte was injected or removed using a capillary located in the middle of the sandbox at a depth of $15 \mathrm{~cm}$. In all cases, the source is dipolar and the dipole moment is proportional to the drop in charge density across the inlet/outlet of the capillary. Source localization algorithms based on the wavelet analysis of potential fields and the cross-correlation method were able to determine the position of the source in the sandbox. This opens very promising perspectives to use self-potential information to monitor continuously fracturing processes in a volcanic edifice and to monitor faults, glaciers, and Earth dams just to cite few examples. The next step will concern the development of a finer model of fracturing and diffusion of electromagnetic disturbances away from the source and the development of algorithms that can be used to retrieve the position of the source in the diffusive limit of the Maxwell equations including the distribution of the electrical resistivity in the inverse problem.

\section{Appendix A}

[48] In this appendix, we model analytically the selfpotential response associated with the pulse injection or 
pumping of water in the controlled sandbox. As all the boundaries of the tank are insulating, the electrical potential obeys $\mathbf{n} \cdot \nabla \psi=0$ on these boundaries, where $\mathbf{n}$ is the outward normal unit vector. We note $S$ the surface area of the cross section of the inlet/outlet of the capillary. The electrical conductivity and the streaming potential coupling coefficient of the electrolyte inside the capillary are $\sigma_{i}=$ $\sigma_{f}$ and $C_{i}=0$, respectively, where the subscript $i$ denotes the interior of the capillary. We introduce the effective potential $\Psi_{i}=\psi_{i}$ and $\Psi_{e}=\psi_{e}-C p$ where $\psi_{i}$ and $\psi_{e}$ are the electrical potentials inside and outside the ellipsoidal inclusion, respectively. The streaming potential current density is given by,

$$
\mathbf{J}_{S}=\left\{\begin{array}{c}
-\sigma_{f} C \nabla p, \text { on } \partial \Omega \\
0, \text { outside } \partial \Omega
\end{array}\right.
$$

where $\partial \Omega$ denotes the interface between the capillary and the sand. The corresponding boundary value problem to solve is given by the solution of Laplace equation $\nabla^{2} \Psi=0$ supplemented by boundary conditions that ensures the continuity of the potential $\Psi$ and the normal component of the total current density $\mathbf{J}$ at the boundary $\partial \Omega$. Using the approach developed by Fitterman [1979, 1981] and Fedorov et al. [2001], the analytical solution for the electrical potential at position $\mathrm{P}$ for an infinite space is,

$$
\psi(P)=\frac{S}{\pi}(C \delta p) \frac{\mathbf{e}_{z} \cdot \mathbf{r}}{r^{3}} .
$$

where $\mathbf{e}_{z}$ is the vertical unit vector perpendicular to the surface of the inlet of the capillary and $r=$ $\sqrt{x^{2}+y^{2}+(z-h)^{2}}$ correspond to the distance between the electrode $\mathrm{P}$ and the center of the boundary $\partial \Omega$. Therefore the electrical potential is,

$$
\psi(P)=\frac{S}{\pi}(C \delta p) \frac{z-h}{\left[x^{2}+y^{2}+(z-h)^{2}\right]^{3 / 2}} .
$$

Then, we use the method of images to satisfy the boundary conditions $\mathbf{n} \cdot \nabla \psi=0$ at all the boundaries and we remove the potential at the position of the reference electrode to compare the model to the experimental data.

[49] Acknowledgments. This work is supported by the French National Research Council (CNRS), the GDR-FORPRO (Research Action number 2005.II), ANR-Project POLARIS, and the French National Agency for Radioactive Waste Management (ANDRA). Joël Lancelot is strongly thanked for his support through the GDR FORPRO. This is GDR-FORPRO contribution 2007/05 A. S. Byrdina is supported by E2C2, A Specific Targeted Project of the European Community. We thank Stephen Park and two anonymous referees for their very constructive comments. AR thanks Terry Young for his support at CSM.

\section{References}

Block, G. I., and J. G. Harris (2006), Conductivity dependence of seismoelectric wave phenomena in fluid-saturated sediments, J. Geophys. Res., 111, B01304, doi:10.1029/2005JB003798.

Bolève, A., A. Crespy, A. Revil, F. Janod, and J. L. Mattiuzzo (2007), Streaming potentials of granular media: Influence of the Dukhin and Reynolds numbers, J. Geophys. Res., 112, B08204, doi:10.1029/ 2006JB004673.

Butler, D. K., J. L. Llopis, T. L. Dobecki, M. J. Wilt, R. F. Corwin, and G. Olhoeft (1990), Comprehensive geophysical investigation of an existing dam foundation. (Part 2), The Leading Edge, 9, 44-53.
Byrdina, S., S. Friedel, J. Wassermann, and J. Zlotnicki (2003), Self-potential variations associated with ultra-long period seismic signals at Merapi Volcano, Geophys. Res. Lett., 30(22), 2156, doi:10.1029/2003GL018272.

Chandler, R. N. (1981), Transient streaming potential measurements on fluid-saturated porous structures: An experimental verification of Biot's slow wave in the quasi-static limit, J. Acoust. Soc. Am., 70, 116-121.

Crespy, A., A. Bolève, and A. Revil (2007), Influence of the Dukhin and Reynolds numbers on the apparent zeta potential of granular media, J. Colloid Interface Sci., 305, 188-194.

Dea, J. Y., C. I. Richman, and W. M. Boerner (1991), Observation of seismo-electromagnetic earthquake precursor radiation signatures along Southern Californian fault zones: Evidence of long-distance precursor ULF signals observed before a moderate Southern California earthquake episode, Can. J. Phys., 69, 1138-1145.

Fedi, M., and T. Quarta (1998), Wavelet analysis for the regional-residual and local separation of potential field anomalies, Geophys. Prospect. 46(5), 507-525, doi:10.1046/j.1365-2478.1998.00105.

Fedorov, E., V. Pilipenko, and S. Uyeda (2001), Electric and magnetic fields generated by electrokinetic processes in a conductive crust, Phys. Chem. Earth, 26(10-12), 793-799.

Fenoglio, M. A., M. J. S. Johnston, and J. D. Byerlee (1995), Magnetic and electric fields associated with changes in high pore pressure in fault zones: Application to the Loma Prieta ULF emissions, J. Geophys. Res., 100, 12,951-12,958.

Finizola, A., J. F. Lénat, O. Macedo, D. Ramos, J. C. Thouret, and F. Sortino (2004), Fluid circulation and structural discontinuities inside Misti volcano (Peru) inferred from self-potential measurements, J. Volcanol. Geotherm. Res., 135(4), 343-360.

Finizola, A., A. Revil, E. Rizzo, B. Angeletti, S. Piscitelli, J. Morin, L. Mocochain, and F. Sortino (2006), Hydrogeological insights at Stromboli volcano (Italy) from geoelectrical, temperature, and $\mathrm{CO}_{2}$ soil degassing investigations, Geophys. Res. Lett., 33, L17304, doi:10.1029/2006GL026842.

Fitterman, D. V. (1979), Theory of electrokinetic-magnetic anomalies in a faulted half-space, J. Geophys. Res., 84, 6031-6041.

Fitterman, D. V. (1981), Correction to Theory of electrokinetic-magnetic anomalies in a faulted half-space, J. Geophys. Res., 86, 9585-9588.

Fraser-Smith, A. C., A. Bernardi, P. R. McGill, M. E. Ladd, R. A. Helliwell, and O. G. Villard Jr. (1990), Low frequency magnetic field measurements near the epicenter of the Ms 7.1 Loma Prieta earthquake, Geophys. Res. Lett., 17, 1465-1468.

Freund, F., M. A. Salgueiro da Silva, B. W. S. Lau, A. Takeuchi, and H. H. Jones (2006a), Electric currents along earthquake faults and the magnetization of pseudotachylite veins, Tectonophysics.

Freund, F., A. Takeuchi, and B. W. S. Lau (2006b), Electric currents streaming out of stressed igneous rocks. A step toward understanding pre-earthquake low frequency EM emissions, Phys. Chem. Earth, 31, 389-396.

Gaffet, S., Y. Guglielmi, J. Virieux, G. Waysand, A. Chwala, R. Stolz, C. Emblanch, M. Auguste, D. Boyer, and A. Cavaillou (2003), Simultaneous seismic and magnetic measurements in the Low-Noise Underground Laboratory (LSBB) of Rustrel, France, during the 2001 January 26 Indian earthquake, Geophys. J. Int., 155, 981-990.

Garon, M., A. Légaré, R. Guardo, P. Savard, and M. D. Buschmann (2002), Streaming potentials maps are spatially resolved indicators of amplitude, frequency and ionic strength dependant responses of articular cartilage to load, J. Biomechanics, 35(2), 207-216.

Gex, P. (1980), Electrofiltration phenomena associated with several dam sites, Bull. Soc. Vaud Sci. Nature, 357(75), 39-50.

Gouy, G. (1910), About the electric charge on the surface of an electrolyte, J. Phys. A, 9, 457-468.

Grant, F. S., and G. F. West (1965), Interpretation Theory in Applied Geophysics, McGraw-Hill Book.

Haartsen, M. W., and M. N. Toksöz (1996), Dynamic streaming currents from seismic point sources in homogeneous poroelastic media, Geophys. J. Int., 132, 256-274.

Holschneider, M. (1995), Wavelets: An Analysis Tool, Oxford Univ. Press. Ishido, T. (1981), Streaming potential associated with hydrothermal convection in the crust: A possible mechanism of self-potential anomalies in geothermal areas (in Jap., with Engl. Abstr.), J. Geotherm. Res. Soc. Jpn., $3,87-100$.

Ishido, T. (1989), Self-potential generation by subsurface water flow through electrokinetic coupling, in Detection of Subsurface Flow Phenomena, Lecture Notes in Earth Sciences, 27, edited by G.-P. Merkler et al., $121-131$, Springer-Verlag, New York.

Ishido, T., and M. Mizutani (1981), Experimental and theoretical basis of electrokinetic phenomena in rock-water systems and its application to geophysics, J. Geophys. Res., 86, $1763-1774$. 
Iuliano, T., P. Mauriello, and D. Patella (2002), Looking inside Mount Vesuvius by potential fields integrated probability tomographies, J. Volcanol. Geotherm. Res., 113, 363-378.

Jardani, A., J. P. Dupont, and A. Revil (2006), Self-potential signals associated with preferential ground water flow pathways in sinkholes, J. Geophys. Res., 111, B09204, doi:10.1029/2005JB004231.

Kulessa, B., B. Hubbard, G. H. Brown, and J. Becker (2003), Earth tide forcing of glacier drainage, Geophys. Res. Lett., 30(1), 1011 , doi:10.1029/2002GL015303.

Leake, I. S. A., and P. A. Hsieh (1997), Simulation of deformation of sediments from decline of ground-water levels in an aquifer underlain by a bedrock step, U.S. Geological Survey Open File report 97-47.

Leroy, P., and A. Revil (2004), A triple layer model of the surface electrochemical properties of clay minerals, J. Colloid Interface Sci., 270(2), $371-380$.

Leroy, P., A. Revil, S. Altmann, and C. Tournassat (2007), Composition of the intersticial pore water of charged porous media. Theory and application to the Callovo-Oxfordian argilite, Geochim. Cosmochim. Acta, 71(5), 1087-1097, doi:10.1016/j.gca.2006.11.009

Linde, N., D. Jougnot, A. Revil, S. Matthäi, T. Arora, D. Renard, and C. Doussan (2007), Streaming current generation in two-phase flow conditions, Geophys. Res. Lett., 34(3), L03306, doi:10.1029/2006GL028878.

Lorne, B., F. Perrier, and J. P. Avouac (1999), Streaming potential measurements 1. Properties of the electrical double layer from crushed rock samples, J. Geophys. Res., 104, 17,857-17,877.

Maineult, A., Y. Bernabé, and P. Ackerer (2005), Detection of advected concentration and $\mathrm{pH}$ fronts from self-potential measurements, J. Geophys. Res., 110(B11), B11205, doi:10.1029/2005JB003824.

Maineult, A., Y. Bernabé, and P. Ackerer (2006a), Detection of advected concentration and $\mathrm{pH}$ fronts from self-potential measurements, J. Geophys. Res., 110(B11), B11205, doi:10.1029/2005JB003824.

Maineult, A., Y. Bernabé, and P. Ackerer (2006b), Detection of advected, reacting redox fronts from self-potential measurements, J. Contam. Hydrol., 86(1-2), 32-52.

Mallat, S. (1999), A Wavelet Tour of Signal Processing, Academic Press.

Merkler, G. P., H. Armbruster, H. Hötzl, P. Marshall, A. Kassel, and E. Ungar (1989), Modelling of streaming potentials and thermometrical measurements at a big laboratory channel, in Lecture Notes in Earth Sciences, Vol. 27, Edited by G. P. Merkler et al., Detection of Subsurface Flow Phenomena, Springer-Verlag Berlin Heidelberg.

Minsley, B. J., J. Sogade, and F. D. Morgan (2007), Three-dimensional source inversion of self-potential data, J. Geophys. Res., 112, B02202, doi:10.1029/2006JB004262.

Moore, J. R., and S. D. Glaser (2006), The origins of the self-potential response during hydraulic fracturing, Eos Trans. $A G U, 87(15)$, Fall Meet. Suppl., Abstract NS21A-04.

Moore, J. R., and S. D. Glaser (2007), Self-potential observations during hydraulic fracturing, J. Geophys. Res., 112, B02204, doi:10.1029/ 2006JB004373.

Moore, J. R., S. D. Glaser, H. F. Morrison, and G. M. Hoversten (2004), The streaming potential of liquid carbone dioxide in Berea sandstone, Geophys. Res. Lett., 31(17), L17610, doi:10.1029/2004GL020774.

Murty, B. V. S., and P. Haricharan (1985), Nomogram for the complete interpretation of spontaneous polarization profiles and sheet like and cylindrical two-dimensional sources, Geophysics, 50, 1127-1135.

Nitsan, U. (1977), Electromagnetic emission accompanying fracture of quartz-bearing rocks, Geophys. Res., Lett., 4, 333-336.

Olhoeft, G. R. (1985), Low-frequency electrical properties, Geophysics, 50, 2492-2503

Palciauskas, V. V., and P. A. Domenico (1989), Fluid pressures in deforming porous rocks, Water Resour. Res., 25, 203-213.

Park, S. K., M. J. S. Johnston, T. R. Madden, F. D. Morgan, and H. F. Morrison (1993), Electromagnetic precursors to earthquakes in the ULF band: A review of observations and mechanisms, Rev. Geophys., 31(2), 117-132, 1029/93RG00820.

Park, S. K., W. Dalrymple, and J. C. Larsen (2007), The 2004 Parkfield earthquake: Test of the electromagnetic precursor hypothesis, J. Geophys. Res., 112, B05302, doi:10.1029/2005JB004196.

Paul, M. K. (1965), Direct interpretation of self-potential anomalies caused by inclined sheets of infinite horizontal extensions, Geophysics, 30, 418423.

Pengra, D., and P. Wong (1995), Pore size, permeability and electrokinetic phenomena, in Access in Nanoporous Materials, edited by T. J. Pinnavaia and M. F. Tho, pp. 295 - 317, Springer, New York.

Perrier, F., and P. Morat (2000), Characterization of electrical daily variations induced by capillary flow in the non-saturated zone, Pure Appl. Geophys., 157, 785-810.

Perrier, F., et al. (1997), A one-year systematic study of electrodes for long period measurements of the electric field in geophysical environments, J. Geomag. Geoelectr., 49, 1677-1696.
Perrier, F., M. Trique, B. Lorne, J.-P. Avouac, S. Hautot, and P. Tarits (1998), Electrical potential variations associated with yearly lake level variations, Geophys. Res. Lett., 25, 1955-1959.

Rao, A. D., and R. H. V. Babu (1984), Quantitative interpretation of selfpotential anomalies due to two-dimensional sheet like bodies, Geophy sics, 48, 1659-1664

Revil, A. (2007), Thermodynamics of transport of ions and water in charged and deformable porous media, J. Colloid Interface Sci., 307(1), 254-264

Revil, A., and P. Leroy (2001), Hydroelectric coupling in a clayey material, Geophys. Res. Lett., 28(8), 1643-1646.

Revil, A., and N. Linde (2006), Chemico-electromechanical coupling in microporous media, J. Coll. Interf. Sci., 302, 682-694.

Revil, A., and P. A. Pezard (1998), Streaming potential anomaly along faults in geothermal areas, Geophys. Res. Lett., 25(16), 3197-3200.

Revil, A., P. A. Pezard, and P. W. J. Glover (1999a), Streaming potential in porous media. 1. Theory of the zeta-potential, J. Geophys. Res., 104(B9), $20,021-20,031$

Revil, A., H. Schwaeger, L. M. Cathles, and P. Manhardt (1999b), Streaming potential in porous media. 2. Theory and application to geothermal systems, J. Geophys. Res., 104(B9), 20,033-20,048.

Revil, A., L. Ehouarne, and E. Thyreault (2001), Tomography of selfpotential anomalies of electrochemical nature, Geophys. Res. Lett., 28(23), 4363-4366.

Revil, A., V. Naudet, J. Nouzaret, and M. Pessel (2003), Principles of electrography applied to self-potential electrokinetic sources and hydrogeological applications, Water Resour. Res., 39(5), 1114, doi:10.1029/ 2001 WR000916.

Revil, A., G. Saracco, and P. Labazuy (2003), The volcano-electric effect, J. Geophys. Res., 108(B5), 2251, doi:10.1029/2002JB001835.

Revil, A. A., F. Finizola, and M. Sortino (2004), Geophysical investigations at Stromboli volcano, Italy. Implications for ground water flow and paroxysmal activity, Geophys. J. Int., 157(1), 426-440.

Revil, A., P. Leroy, and K. Titov (2005), Characterization of transport properties of argillaceous sediments. Application to the Callovo-Oxfordian Argillite, J. Geophys. Res., 110, B06202, doi:10.1029/2004JB003442.

Rizzo, E., B. Suski, A. Revil, S. Straface, and S. Troisi (2004), Self-potential signals associated with pumping-tests experiments, J. Geophys. Res., 109, B10203, doi:10.1029/2004JB003049.

Sheffer, M. R., and J. A. Howie (2003), A numerical modelling procedure for the study of the streaming potential phenomenon in embankment dams, Symposium on the Application of Geophysics to Engineering and Environmental Problems, San Antonio, pp. 475-487.

Sill, W. R. (1983), Self-potential modeling from primary flows, Geophysics, $48,76-86$

Soloviev, S. P., and J. J. Sweeney (2005), Generation of electric and magnetic field during detonation of high explosive charges in boreholes, J. Geophys. Res., 110, B01312, doi:10.1029/2004JB003223.

Surkov, V. V., and V. A. Pilipenko (1997), Magnetic effects due to earthquakes and underground explosions: A review, Ann. Geofis., XL, 1-13.

Suski, B., E. Rizzo, and A. Revil (2004), A sandbox experiment of selfpotential signals associated with a pumping-test, Vadose Zone J., 3 , $1193-1199$.

Suski, B., A. Revil, P. Konosavsky, C. Dagès, M. Voltz, and O. Huttel (2006), Monitoring of an infiltration experiment using the self-potential method, Water Resour. Res., 42, W08418, doi:10.1029/2005WR004840. Szymczyk, A., P. Fievet, and A. Foissy (2002), Electrokinetic characterization of porous plugs from streaming potential coupled with electrical resistance measurements, J. Colloid and Interface Science, 255, $323-$ 331, doi:10.1006/jcis.2002.8591

Tate, J., and W. Daily (1989), Evidence of electro-seismic phenomena, Phys. Earth Planet. Inter., 57, 1-10.

Titov, K., Loukhmanov, and A. Potapov (2000), Monitoring of water seepage from a reservoir using resistivity and self-polarization methods: case history of the Petergoph fountain water supply system, First Break, 18 , $431-435$

Triumf, C. A., and H. Thunehed (1996), Two years of Self-Potential measurements on a large dam in northern Sweden. Procs. Repair and Upgrading of Dams, KTH, Stockholm: 307- 315

Ushijima, K., H. Mizunaga, and T. Tanaka (1999), Reservoir monitoring by a 4-D electrical technique, The Leading Edge, 12, 1422-1424.

Wang, H. F. (2000), Theory of Linear Poroelasticity with Applications to Geomechanics and Hydrogeology, Princeton Univ. Press, Princeton, $287 \mathrm{pp}$.

Wilt, M. J., and R. F. Corwin (1989), Numerical modeling of self-potential anomalies due to leaky dams: Model and field examples. In: Lecture Notes in Earth Sciences, vol. 27, ed. G.P. Merkler et al., Detection of subsurface flow phenomena, Springer-Verlag, Berlin Heidelberg: 73-89. Yasukawa, K., E. Kusdinar, and H. Muraoka (2002), Reservoir response to a well test identified through a self-potential monitoring at the $\mathrm{Ma}$ - 
taloko geothermal field, central Flores, Indonesia, Geol. Surv. Japan, 53, $355-363$.

Yoshida, S. (2001), Convection current generated prior to rupture in saturated rocks, J. Geophys. Res., 106, 2103-2120.

Yoshida, S., and T. Ogawa (2004), Electromagnetic emissions from dry and wet granite associated with acoustic emissions, J. Geophys. Res., 109, B09204, doi:10.1029/2004JB003092.

Yoshida, S., P. Manjgaladze, D. Zilpimiani, M. Ohnaka, and N. Nakatani (1994), Electromagnetic emissions associated with frictional sliding of rock, in Electromagnetic Phenomena Related to Earthquake Prediction, edited by M. Hayakawa and Y. Fujinawa, 307-322, Terrapub, Tokyo.
Yoshida, S., M. Uyeshima, and N. Nakatani (1997), Electric potential changes associated with slip failure of granite: preseismic and coseismic signals, J. Geophys. Res., 102, 14,883-14,897.

A. Bolève, SOBESOL, Savoie Technolac, BP 230, F-73375 Le Bourget du Lac Cedex, France.

S. Byrdina, IPGP, 4 Place Jussieu, Paris, France

A. Crespy, CNRS-CEREGE, Université Aix Marseille III, Aix en Provence, France.

P. Henry, Collège de France, Université Aix Marseille III, France.

A. Jardani and A. Revil, Colorado School of Mines, Department of Geophysics, Golden, Colorado, USA. (arevil@mines.edu)

N. Linde, Swiss Federal Institute of Technology, Institute of Geophysics, Zurich, Switzerland. 